\title{
On Dynamically Consistent Eddy Fluxes
}

\author{
Pavel S. Berloff
}

Woods Hole Oceanographic Institution, USA

and

Department of Applied Mathematics and Theoretical Physics, University of Cambridge, UK

September, 2004

Accepted: Dynamics of Atmospheres and Oceans

Corresponding author e-mail and address: pberloff@whoi.edu;

DAMTP-CMS, University of Cambridge, Wilberforce Rd., Cambridge, CB3 0WA, UK. 


\begin{abstract}
The role of mesoscale oceanic eddies in driving the large-scale currents is studied in an eddy-resolving, double-gyre ocean model. The new diagnostic method is proposed, which is based on dynamical decomposition of the flow into the large-scale and eddy components. The method yields the time history of the eddy forcing, which can be used as additional, external forcing in the corresponding non-eddy-resolving model of the gyres. The main strength of this approach is in its dynamical consistency: the non-eddy-resolving solution driven by the eddy forcing history correctly approximates the original large-scale flow component. It is shown that statistical decompositions, which are based on space-time filtering diagnostics, are dynamically inconsistent. The diagnostics algorithm is formulated and tested, and the diagnosed eddies are analysed, both statistically and dynamically. It is argued that the main dynamic role of the eddies is to maintain the eastward-jet extension of the subtropical western boundary current. This is done largely by both the time-mean isopycnal-thickness flux and the relativevorticity eddy flux fluctuations. The fluctuations drive large-scale flow through the nonlinear rectification mechanism. The relative-vorticity flux contributes mostly to the eastward jet meandering. Finally, eddy fluxes driven by both the eddies and the large-scale flow are found to be important. The latter is typically neglected in the analysis, but here it corresponds to important large-scale feedback on the eddies.
\end{abstract}




\section{Introduction}

The need to understand the large-scale/eddy interactions and to account for them in Oceanic General Circulation Models (OGCMs) is one of the fundamental challenges in physical oceanography. It is widely recognised that, along with surface forcing, the mesoscale eddy fluxes of momentum and potential vorticity (PV) are capable of driving the large-scale currents. In the OGCMs, the eddy fluxes are partially resolved but mostly parameterised in terms of simple mathematical models. It is difficult to test these simple models, because it is difficult to observe the real eddy fluxes. At this point, a promising strategy is to study eddy fluxes in idealised, eddy-resolving ocean models, as it is done in this paper.

In the introduction, the background literature is discussed, the problem is posed, and the ocean models and the eddy-resolving solution are described. The new method of flow decomposition into the large-scale and eddy components is given in section 2. Analysis and interpretation of the corresponding eddy fluxes is in section 3, which is followed by the summary.

\subsection{Background}

The role of eddies in the midlatitude ocean gyres remains poorly understood. Early theoretical works on the gyres consider simple and steady, linear (Stommel 1948; Munk 1950) and nonlinear (Fofonoff 1954; Moore 1963) analytic solutions of the barotropic vorticity equation. Most of the recent theoretical advances, which focus on more realistic circulation regimes, are made with the help of numerical models. In particular, it is shown that the gyres have multiple steady states (e.g., Cessi and Ierley 1995; Speich et al. 1995; Sheremet et al. 1997; Berloff and Meacham 1998), all of which are unstable for Reynolds number (Re) larger than

its critical value. At supercritical, but still significantly smaller than the oceanic, Re, the 
gyres operate in dynamically complex turbulent regimes, which are, generally, even more realistic. The main aspects of the wind-driven midlatitude ocean gyres are the broad and slow currents in the interior of the basin, the narrow and swift western boundary currents (WBCs) with their eastward jet (EJ) extensions and adjacent recirculation zones, and the mesoscale eddies generated by flow instabilities and nonlinear dynamics. The eddies are typically defined as fluctuations around the time-mean background flow. Nonlinear interactions between the eddies and the background flow are complex (e.g., Bryan 1963; Veronis 1966; Holland 1978; Haidvogel et al. 1992; Berloff and McWilliams 1999a,b), and the role of eddies increases with Re (e.g., Siegel et al. 2001). Importance of the large-scale advection in driving the recirculations surrounding the main EJ by supplying PV anomalies is argued in Cessi et al. (1987), and the role of the time-mean eddy/eddy PV flux is studied in Jayne et al. (1996).

Modelling eddy effects by simple mathematical models is a classical turbulence problem, which is particularly difficult in the ocean, where spatially dense and temporally long observations of the eddy field are problematic. The most common model of this kind is turbulent diffusion, which represents unresolved eddies in virtually all OGCMs. The basic assumption of the diffusion model is that the large-scale currents are smoothed out rather than enhanced by the eddies, but there are examples of the anti-diffusion phenomenon (Starr 1968). It is argued, that this phenomenon - impossible in the classical turbulence scenario - can be understood in terms of the long-range transport of momentum or vorticity, that is associated with their generation in one place but dissipation in another one (McIntyre 2000). In the ocean this transport is done by the eddy fluxes, and the necessary condition for them to be approximated by the diffusion is their down-gradient orientation with respect to the background gradient of the fluxed quantity. Different orientations of the eddy fluxes are found in idealised eddy- 
resolving models (e.g., Treguier 1999; Jayne et al. 1996; Jayne and Hogg 1999; Drijfhout and Hazeleger 2001; Roberts and Marshall 2000), therefore, relevance of the diffusion approach is uncertain, and alternative approaches have to be looked for (e.g., Berloff 2004).

\subsection{Statement of the Problem}

Solving for the ocean mesoscale-turbulence problem requires: (i) efficient diagnostics of the eddies, (ii) suitable mathematical model for the eddy effects, and (iii) relationship between parameters of this model and the large-scale flow. This paper deals with the eddy diagnostics and its interpretation; the mathematical model based on these results is in Berloff (2004); and the search for parameter relationships is a matter of the future. The problem is addressed in the context of the idealised ocean circulation, but the method and the ideas can be applied to other geophysical and turbulent flows.

The starting question is: What is a meaningful decomposition of the flow solution into the large-scale and eddy components? Obviously, the flow decomposition is not unique, and in physical oceanography the most common approach is to decompose any flow into the infinitetime average, representing the large-scale component, and fluctuations around this average, representing the eddies. Alternatively, large-scale flow can be filtered out by a more general space-time filter. Here, a new idea of flow decomposition, and, therefore, a new definition of the eddy fluxes is proposed and implemented. According to this idea, the large-scale flow is filtered out of the original data interactively, as the solution of a non-eddy-resolving model integrated in time and corrected by the interactively calculated eddy forcing. Thus, the noneddy-resolving model acts as the dynamical filter. The main strength of this approach is in its dynamical consistency: the non-eddy-resolving solution driven by the eddy forcing history correctly approximates the original flow. The practical significance of the approach is in the 
idea that the dynamically consistent eddy forcing can be modelled as a random process (Berloff 2004). Once the flow is decomposed, the following questions are asked. What is the largescale dynamical effect of the eddies? What are the principal mechanisms involved? What are relative contributions of different eddy flux components? Can the eddy effects be represented as a diffusion process?

Although the dynamical decomposition method is essentially a diagnostic tool that requires detailed knowledge of the flow, it prepares solid ground for advance toward "parameterisation" of the eddy effects. After mathematical model of the eddy effects is developed and its parameters are related to the large-scale flow, eddy-resolving simulations will not be required for predicting the large-scale flow statistics. An important step toward this goal is to study sensitivity of the large-scale response to different simplifications of the eddy effects obtained in the dynamically consistent way (Berloff 2004).

\subsection{Ocean Models}

Here, the Eddy-Resolving (ER) model is used to obtain the reference solution, which contains both the large-scale and eddy components. The model represents midlatitude ocean, with a prescribed density stratification, and in in a flat-bottom square basin with north-south and east-west boundaries. The QG PV equations (Pedlosky 1987) for $N$ dynamically active isopycnal layers are:

$$
\frac{\partial q_{i}}{\partial t}+J\left(\psi_{i}, q_{i}\right)+\beta \frac{\partial \psi_{i}}{\partial x}=\frac{\delta_{i, 1}}{\rho_{1} H_{1}} \nabla \times \tau+\nu \nabla^{4} \psi_{i}
$$

where $\delta_{i, j}=1$ if $i=j$, and $\delta_{i, j}=0$ if $i \neq j$, and $i$ is the layer index starting from the top.

The meridional planetary vorticity gradient is $\beta$, the surface wind stress is $\tau$, and $J($,$) is the$ Jacobian operator. The PV anomalies, $q_{i}$, are connected with the velocity streamfunctions, 
$\psi_{i}$, through the coupled elliptic equations:

$$
\nabla^{2} \psi_{i}-\left(1-\delta_{i, 1}\right) S_{i, 1}\left(\psi_{i}-\psi_{i-1}\right)-\left(1-\delta_{i, N}\right) S_{i, 2}\left(\psi_{i}-\psi_{i+1}\right)=q_{i}
$$

with the stratification parameters $S_{i, 1}$ and $S_{i, 2}$. The horizontal velocity components are found as

$$
u_{i}=-\frac{\partial \psi_{i}}{\partial y}, \quad v_{i}=\frac{\partial \psi_{i}}{\partial x}
$$

and on the lateral boundaries velocity is zero. Also, there is the mass conservation constraint for each layer:

$$
\frac{\partial}{\partial t} \iint \psi_{i}(x, y) d x d y=0
$$

Parameters of the model and basic features of the flow solution (Fig. 1) are discussed in appendix A. After the initial spin-up from the state of rest, the solution equilibrates statistically. Then, it is computed for $10^{4}$ days and stored in terms of $\psi$ for the analysis on the coarsened, $129 \times 129$ grid, with 1-day time intervals. This data set is referred to as the coarse-grained (or, projected on the coarse grid), reference ER solution. In general, the coarse-graining method can be altered by using a coarse-gridscale filter, but here the simplest option is used, which is equivalent to the common observational practice of estimating point-wise quantities. Also, the filtering would result in underestimating the variance of the flow fluctuations. The coarsegraining projection can be more formally expressed as

$$
\widetilde{\psi}(T, \mathbf{X}) \equiv \operatorname{Proj}[\psi(t, \mathbf{x})] \equiv \int_{X-\Delta X / 2}^{X+\Delta X / 2} \int_{Y-\Delta Y / 2}^{Y+\Delta Y / 2} \int_{T-\Delta T / 2}^{T+\Delta T / 2} K(t, \mathbf{x} ; T, \mathbf{X}) \psi(t, \mathbf{x}) d x d y d t
$$

In this paper, the projection kernel is chosen to be a simple Dirac delta function,

$$
K(t, \mathbf{x} ; T, \mathbf{X}) \psi(t, \mathbf{x})=\delta(X-x) \delta(Y-y) \delta(T-t)
$$


for each isopycnal layer. A more general form of the kernel allows for heterogeneous averaging near boundaries and nested grids. Further below, tilde over $\psi$ is omitted for simplicity, unless otherwise stated.

How crucial is the use of the ER model for the dynamically consistent eddy flux analysis? First, this analysis can be viewed as a purely diagnostic tool for solutions of any comprehensive ER model. Second, there is a hope that the diagnosed eddy effects will be "parameterised", hence resolving the eddies will not be required for modelling the large-scale flow. However, before this is achieved, (a) generic properties of the eddy fluxes have to be sorted out and understood, (b) a model of the eddy effects has to be put forward, and (c) relationships between the model parameters and the large-scale flow patterns have to be established. Finally, the ER solution can be, potentially, replaced with interactive ocean observations, and the associated coarse-grid model can be used for the analysis of the observations.

The large-scale and eddy components of the flow, arbitrary so far, are denoted with overbar and prime, respectively, and the full flow is:

$$
\mathbf{u}(t, \mathbf{x})=\overline{\mathbf{u}}+\mathbf{u}^{\prime}, \quad q(t, \mathbf{x})=\bar{q}+q^{\prime} .
$$

The eddy forcing is defined as

$$
f=J(\bar{\psi}, \bar{q})-J(\psi, q)=\nabla \cdot \overline{\mathbf{u}} \bar{q}-\nabla \cdot \mathbf{u} q=-\nabla \cdot \mathbf{F}^{\prime}
$$

where $\mathbf{F}^{\prime}$ is the eddy flux of the PV. In the simple form, the eddy forcing incorporates eddy effects, which are otherwise missing from the non-ER dynamics. Note, that the eddy forcing can be defined for any decomposition (7), but in the dynamical decomposition method the large-scale flow component is unambiguously defined as the solution of the non-ER model interactively corrected by the corresponding eddy forcing (8). 
The ER and non-ER models have the same general set-up, but the former has far fewer degrees of freedom and solves only for the large-scale flow evolution. The governing non-ER PV equations are:

$$
\frac{\partial \overline{q_{i}}}{\partial T}+\overline{u_{i}} \frac{\partial \overline{q_{i}}}{\partial X}+\overline{v_{i}} \frac{\partial \overline{q_{i}}}{\partial Y}+\beta \frac{\partial \overline{\psi_{i}}}{\partial X}=\frac{\delta_{i, 1}}{\rho_{1} H_{1}} \nabla \times \tau+\bar{\nu} \nabla^{4} \overline{\psi_{i}}+f_{i},
$$

where $(X, Y)$ are the coarse-grid space coordinates, $\overline{q_{i}}$ and $\overline{\psi_{i}}$ are the non-ER (i.e., large-scale) PV anomaly and velocity streamfunction, and all of the derivatives are calculated on the coarse grid. Using different time scale, $T$, indicates that the non-ER model allows for the larger time steps due to the relaxed Courant condition (2.4 hours time step is used). The elliptic equation (2) is similarly modified. In (9) $f_{i}$ is the eddy forcing, and the eddy diffusivity enhancement, $\bar{\nu}-\nu$, parameterises the sub-mesoscale eddy forcing wiped out as a result of the coarse-grid projection of the ER solution (appendix A). Here, $\bar{\nu}=10 \nu=10^{3} \mathrm{~m}^{2} \mathrm{~s}^{-1}$, and sensitivity study with the values of $\bar{\nu}$, which are 10 times smaller or larger than that, confirms all of the basic conclusions of this paper. This is so, because most of the dissipation occurs near the western boundary and the dynamically important eddy fluxes in the eastward-jet region are not very sensitive to the diffusivity enhancement. Given that the coarse grid has 4 times less points in each direction, the grid-scale Reynolds number, $U \Delta / \bar{\nu}$, where $U$ is some typical velocity and $\Delta$ is the grid scale, is reduced by a factor of 0.4 . This is done in order to be able to resolve marginally, by just one point, the Munk scale, $[\bar{\nu} / \beta]^{1 / 3}$. In a more advanced application of the ideas developed in this paper, either $\Delta$ or $\bar{\nu}$ can be spatially inhomogeneous, so that both the grid-scale Reynolds number is kept fixed and the Munk scale is well resolved.

\section{Flow Decomposition into the Large-Scale and Eddy Components}




\subsection{Dynamical Decomposition Method}

This section explains how a non-ER model can be used to decompose the flow. The idea is to introduce the dynamical constraint: Given a non-ER model, the flow decomposition has to be such that the resulting eddy force yields the non-ER solution which correctly approximates the large-scale structure of the original flow. The eddy flux and force based on the decomposition satisfying this constraint are referred to as the dynamically consistent ones. There are three important consequences of the constraint: (i) the eddy forcing becomes a part of the prognostic, dynamic model for the large-scale flow; (ii) the flow decomposition, the eddy flux, and the non-ER model are always defined together; and (iii) the non-ER model equations have to be solved explicitly as a part of the decomposition process. Another, less obvious consequence is that dynamically consistent eddy forcing can be replaced by a random process, and the corresponding non-ER solution will correctly approximate the original flow (Berloff 2004).

The dynamical decomposition algorithm is based on integrating and interactively correcting the non-ER model solution with the information supplied by the structure of the full flow. This information enters the non-ER model in terms of the interactively calculated eddy force, and the eddies are continuously and interactively defined in terms of the structural differences between the full and non-ER flows. More specifically, a non-ER model is initialised with the initial conditions for the ER flow and integrated in time. At each moment of time, the largescale component of the flow, $\bar{\psi}$, is defined as the non-ER solution; and the eddy component, $\psi^{\prime}$, is found as the difference between the full flow and the non-ER solution. In each isopycnal layer, at each grid point, and on each time step, the instantaneous eddy forcing, $f(t, \mathbf{x}$, is found according to $(8)$, where $J($,$) and \nabla$ are the coarse-grid operators. Thus, $f(t, \mathbf{x}$ incorporates information from both the non-ER and ER solutions. Next, the non-ER model is stepped 
forward with this eddy forcing term and the process is repeated for the next time step. In the end, the non-ER solution is obtained over the same time interval as the ER reference solution. To summarize, the eddies are literally the unresolved fluctuations of the non-ER dynamics, but, nevertheless, they are accounted for in terms of the (residual) eddy forcing.

Decomposing turbulence by dynamic models is not common. An alternative method, applied to the large-eddy simulation of the isotropic turbulence, calculates the eddy forcing by making use of the truncated approximation of the Navier-Stokes equation solved on a finer grid (Domaradzki et al. 2002). This method has no enhanced eddy diffusivity, therefore the energy gradually piles up at small scales, and to avoid problem the fine-grid flow has to be periodically reinitialised with the parallel coarse-grid solution. The dynamical decomposition method is more simple and straightforward, but it is expected to work only when the eddy forcing is a leading-order term. Fortunately, this is the case in the oceanic gyres. Although the method proposed here also involves corrections given by differences between the modelled and reference flows, it is different from the method based on calculating residual force from the tendency term (D'Andrea and Vautard 2000) and from the approach in which the residual force is used to correct the eddy diffusivity coefficient (Kaas et al. 1999). It is also different from the approach in which solution is corrected by the time-mean component rather than the full history of the eddy forcing (Marshall and Molteni 1993). In the present study, an attempt to account for other eddy effects by correcting the diffusion operator and the $\beta$-term destroys the leading-order linear balance in the western-boundary viscous layer and corrupts the integral PV balance (section 2.3). In turn, this induces solution runaway characterised by exaggerated gyres.

Generally speaking, decomposition (7) and the corresponding eddy flux history are not 
unique. For example, $\bar{\psi}$ (or, $\bar{q}$ ) can be statistically filtered out as

$$
\bar{\psi}(t, \mathbf{x})=\iint G\left(t-t^{\prime}, \mathbf{x}-\mathbf{x}^{\prime}\right) \psi\left(t^{\prime}, \mathbf{x}^{\prime}\right) d t^{\prime} d \mathbf{x}^{\prime}
$$

where $G(t, \mathbf{x})$ is a prescribed filtering kernel with unit norm. The main problem with this statistical approach is that the choice of $G(t, \mathbf{x})$ is not unique and physically not constrained. The particular case of (10) that involves averaging in time only is the most common basis for defining the eddy fluxes. Appendix B illustrates dynamical inconsistency of this definition. Thus, it is argued here that the traditional approach should be abandoned or at the very least strongly informed by modelling efforts that focus on parameterising dynamically consistent eddy forcing.

\subsection{Dynamical Decomposition of the Ocean Gyres}

In this section, the dynamical decomposition method is applied to the ER solution.

How well can the non-ER model work without the eddy forcing? In this case, the non-ER model is the coarse-grid model in which truncated dynamic degrees of freedom are simply not accounted for. It is found that the coarse-grid model is not capable of approximating the ER flow, no matter how large or small is the value of $\nu$, because the diffusion model simply can not simulate anti-frictional action of the mesoscale eddies (compare Figs. 2 and 1a,c). With small $\bar{\nu}$, the subtropical WBC generates intense anticyclonic eddies that propagate all the way to the north-western corner of the basin and drive there the time-mean anticyclonic recirculation (Fig. 2a,c). Location of the instantaneous WBC separation point fluctuates strongly, and the time-mean EJ has unrealistically large meridional excursion. This flow regime is formed because the resolved eddies are not efficient in delivering PV from the western boundary into the basin interior. As a result, the PV budget (17) is satisfied by relatively large exchange of 
PV (and mass) between the northern and southern parts of the basin and by the invasion of the subtropical gyre to the cyclonically forced region. Overall, this regime is similar to the free-slip one (Cessi 1991), largely due to the poor resolution of the viscous western boundary layer. The large- $\bar{\nu}$ regime is qualitatively incorrect because it does not have the right EJs: the time-mean eastward flow is broad, slow, and excessively meandering and the WBC eddies are exaggerated. Overall, this flow regime is qualitatively similar to OGCM solutions at moderate Re (e.g., Bryan 1987).

In the ocean gyres, the dynamical decomposition method based on the non-ER model (9) works well (Fig. 3). This is so because the eddy forcing is strong. The time-mean non-ER flow is characterised by the EJs with qualitatively correct shapes, and separating from the boundary in right locations. On the other hand, the non-ER WBCs are broader due to the enhanced diffusivity. In the western part of the basin, which generally is the most vulnerable to under-resolving the eddies, instantaneous non-ER flow smoothly approximates the reference solution (compare Figs. 1c and 3b). In the eastern basin, the eddies are dominated by largescale, small-variance, and relatively fast, westward propagating signal, which is likely generated by instabilities of the subtropical WBC and its EJ extension (Berloff and McWilliams 1999a). The non-ER model overestimates these instabilities, therefore it exaggerates these fluctuations. Success of the non-ER solution in approximating the ER flow is further quantified by significant correlations between the non-ER and ER time series. The basin-integrated potential energy time series have remarkably large correlation coefficient of 0.98. The time-mean kinetic energies differ by factor of 2.5, suggesting that the eddy forcing accounts for most of the eddy activity. On the other hand, the kinetic energy correlation, which is a poorer indicator of the large-scale coherence, is 0.53 . 


\subsection{PV Fluxes and Flux Components}

Given that the ER solution is dynamically decomposed into the large-scale, $\overline{\mathbf{u}}$, and eddy, $\mathbf{u}^{\prime}$ components, different components of PV fluxes can be studied statistically. Each flux component is sum of the time-mean pattern and history of the fluctuations:

$$
\overline{\mathbf{u}}=\langle\overline{\mathbf{u}}\rangle+\{\overline{\mathbf{u}}\}, \quad \mathbf{u}^{\prime}=\left\langle\mathbf{u}^{\prime}\right\rangle+\left\{\mathbf{u}^{\prime}\right\}
$$

denoted by the angular and curly brackets, respectively. In each isopycnal fluid layer, the horizontal PV flux,

$$
\mathbf{F}=\overline{\mathbf{u}} \bar{q}+\overline{\mathbf{u}} q^{\prime}+\mathbf{u}^{\prime} \bar{q}+\mathbf{u}^{\prime} q^{\prime}=\mathbf{F}_{L L}+\mathbf{F}_{L E}^{\prime}+\mathbf{F}_{E L}^{\prime}+\mathbf{F}_{E E}^{\prime}
$$

is the sum of the four scale interaction components, where each term on the left-hand side is denoted by the corresponding term on the right-hand side. Here, the large-scale/large-scale component can be written as

$$
\mathbf{F}_{L L}=\langle\overline{\mathbf{u}}\rangle\langle\bar{q}\rangle+\mathbf{F}_{L L}^{\prime}
$$

where the first term is PV flux driven by the large-scale climatology, and the second term is the resolved, large-eddy flux. The unresolved flux,

$$
\mathbf{F}^{\prime}=\mathbf{F}_{L E}^{\prime}+\mathbf{F}_{E L}^{\prime}+\mathbf{F}_{E E}^{\prime},
$$

is in the main focus of this study. Also, the fluxes are decomposed into the vorticity-type components that represent individual fluxes of relative vorticity (i.e., Reynolds stress divergence):

$$
\mathbf{R}_{i}=\mathbf{u}_{i} \nabla^{2} \psi_{i}
$$

and buoyancy (i.e., the isopycnal-thickness flux):

$$
\mathbf{B}_{i}=\mathbf{u}_{i}\left[-\left(1-\delta_{i, 1}\right) S_{i, 1}\left(\psi_{i}-\psi_{i-1}\right)-\left(1-\delta_{i, N}\right) S_{i, 2}\left(\psi_{i}-\psi_{i+1}\right)\right]
$$


where subscript indicates the isopycnal layer. Here, the fluxes are not decomposed into rotational and divergent components, because this decomposition is not unique in a bounded domain (Fox-Kemper et al. 2003). To summarise, each of the eddy fluxes in (14) can be decomposed into two vorticity-type components and into the time average and fluctuations, thus, there are 12 eddy flux components in total. In contrast, traditional analysis, based on the flow decomposition into the time-mean component and fluctuations, focuses only on 2 fluxes: $\left\langle\mathbf{R}_{E E}^{\prime}\right\rangle$ and $\left\langle\mathbf{B}_{E E}^{\prime}\right\rangle$.

In each isopycnal layer, basin integral of each flux component is zero due to the no-slip boundary condition. Also, due to the mass conservation constraint (4), Stokes theorem, and the no-slip boundary condition:

$$
\frac{\partial}{\partial t} \iint q d x d y=\frac{\partial}{\partial t} \iint \nabla^{2} \psi d x d y=\frac{\partial}{\partial t} \oint_{\Gamma} u_{l} d l=0
$$

where $u_{l}$ is the velocity component along the basin boundary, $\Gamma$. Since $\iint \beta y d x d y=0,(17)$ implies that the basin-integrated absolute $\mathrm{PV}, Q$, is constant, and the rate of $\mathrm{PV}$ generation by the wind is exactly balanced by the diffusive flux of PV through the lateral boundaries:

$$
\iint \nabla \times \tau d x d y=-\nu \iint \nabla^{2} \nabla^{2} \psi d x d y=-\oint_{\Gamma} D_{n} d l
$$

where $D_{n}$ is the diffusive flux component normal to $\Gamma$. In particular, since the deep-ocean isopycnal layers are not exposed to the wind, they have zero integral diffusive fluxes through

the boundaries. In section 3, it is shown that the boundary-generated PV fluxes are essential for eddy effects on the large-scale flow.

\section{Analysis of the Eddy Fluxes}


This section analyses and interprets the dynamically consistent eddy flux and its components, as given by the eddy-resolving model. What flux components (section 2.3) are important and in what way?

Capability of the eddy flux to drive a large-scale flow is easily illustrated for a zonal-jet configuration (Pedlosky 1987). In this case, both positive flux of relative vorticity and positive ageostrophic velocity yield eastward acceleration, and, therefore, drive the eastward jet; and positive divergence of the buoyancy flux yields positive change of depth deviation. In the purely adiabatic and stationary situation, the eddy fluxes produce time-mean meridional circulation with the ageostrophic component that precisely cancels the tendency of the fluxes to alter the time-mean state. This is the wave-mean flow non-acceleration theorem. In the double gyres, the conditions of the theorem are not valid, because there is no zonal symmetry, and the fluiddynamic model is forced (by the wind and through the boundaries) and dissipative. The PV equation allows one to get rid of the ageostrophic velocity component and to formulate the eddy forcing in terms of the flux divergences. Here, since the QG model is determined in terms of the PV, the PV fluxes and their effects on the large-scale flow are considered. As well as the time-mean eddy forcing, purely fluctuating eddy forcing are also capable of driving time-mean large-scale flows through the rectification phenomenon (Haidvogel and Rhines 1983).

\subsection{Full Time-Mean Eddy Fluxes}

The time-mean eddy flux, $\left\langle\mathbf{F}^{\prime}\right\rangle$, is relatively large in the western part of the basin, in the upper ocean, and, particularly, around the subtropical WBC and its EJ. The flux maintains the global PV budget (17) by transporting PV from the boundaries into the basin interior and from one gyre to the other. In the first mechanism, PV generated on the boundary is fluxed through the viscous boundary layer by the diffusion, then it is picked up and fluxed 
farther away by the eddies (Fox-Kemper and Pedlosky 2004). The boundary source of PV is large when the relative vorticity has large horizontal gradient in the off-boundary direction, as it occurs here in the western basin. Eddy fluxes drive large-scale flows by diverging: positive/negative divergence decreases/increases local PV anomaly and, therefore, drives an anticyclonic/cyclonic recirculation. Here, $\left\langle\mathbf{F}^{\prime}\right\rangle$ is strongly divergent around the WBCs and EJs in a similar way to its isopycnal-thickness flux component (Fig. 4f). In the upper ocean, the main positive divergence is to the east of the subtropical WBC and to the south of its EJ extension, and the main negative divergence is to the north of the subtropical EJ - this pattern tends to enhance the EJ. In the deep ocean, the flux is weaker, and around the EJ its divergence pattern is largely opposite to the upper-ocean one. Some characteristics of the cross-EJ eddy flux and its components are summarised in Tables 1 and 2, and discussed further below in terms of their relationship to the time-mean flow gradient (appendix C). Here, some effort is made to simply relate the eddy fluxes and the large-scale flow, or the eddy forcing and its variance, but the results are discouraging. It is obvious that search for such relationships should be based on more suitable mathematical models for the eddy effects (e.g., Berloff 2004).

\subsection{Vorticity-Type Components}

Physical insight into the eddy effects is gained by decomposing the eddy fluxes into the vorticity-type components (section 2.3), $\left\langle\mathbf{R}^{\prime}\right\rangle$ and $\left\langle\mathbf{B}^{\prime}\right\rangle$. Away from the WBCs, the $B^{\prime}$-flux dominates in terms of magnitude and divergence (Fig. 4), which is consistent with some observations ${ }^{1}$. The boundary-generated PV, which is in the relative-vorticity form, is diffusively

\footnotetext{
${ }^{1}$ Below the thermocline, PV eddy flux is dominated by the thickness flux component with divergence that drives cyclonic/anticyclonic recirculation to the north/south of the Gulfstream EJ (Hogg 1993), but there are no conclusive observations in the upper ocean.
} 
fluxed across the viscous sublayer, until it is picked up by the off-shore component of the $R^{\prime}$-flux. In the WBC outside the sublayer, there is a region of intense baroclinic instability (Berloff and McWilliams 1999a). The mesoscale eddies generated over there are characterised by large deviations of the isopycnal surfaces, therefore, they strongly contribute to the thickness flux. Along the EJ, the $R^{\prime}$-flux divergence consists of the sequence of positive and negative anomalies, and the $B^{\prime}$-flux divergence is organised in the well-defined dipole pattern (Fig. 5).

The fluxes substantially force and alter the subtropical WBC: the $B^{\prime}$-divergence slows it down, which is consistent with the local baroclinic instability process, and the $R^{\prime}$-divergence substantially reshapes it. There, the negative $R^{\prime}$-divergence amplifies positive near-boundary vorticity, and, thus, shifts the current axis toward the boundary. Farther away from the boundary, the positive $R^{\prime}$-divergence generates negative PV — this process represents stirring by the eddies that homogenises PV to the east of the jet axis. In more idealised set-up, the mechanism of reorganization of the WBC by the eddies is studied in Berloff and McWilliams 1999b. To the large extent, the WBC separation is controlled by the absolute maximum of the $R^{\prime}$-divergence in the most western, upper-ocean meander of the EJ.

Around the EJ, the $R^{\prime}$-flux does not have simple pattern, unlike its divergence. This is consistent with the barotropic single-gyre solutions (Fox-Kemper and Pedlosky 2004), which have no thickness fluxes. The thickness flux recirculates in the form of the large-scale, cyclonic gyre that covers the WBC/EJ region. This eddy flux recirculation gyre is a part of the global PV exchange between the gyre interiors and the western (and, partially, the southern) boundary, rather than it is a part of the PV (and mass) exchange between the oceanic gyres. The primary dynamic mechanism driving the eddy flux recirculation gyre is baroclinic instability of the WBC that converts boundary-generated relative vorticity into the eddy thickness flux, which 
connects the boundary and the EJ. In sharp contrast with intensive cross-EJ fluxes, the fluxes connecting the subtropical and subpolar gyre are weak (Fig. 4), which is consistent with the inhibited inter-gyre mass exchange (Berloff et al. 2002). This situation is rather different from the one considered in Harrison (1981) and Marshall (1984), because of the no-slip boundary condition and asymmetry of the wind forcing. The $B^{\prime}$-flux crosses the EJ in the form of well-defined, broad, and approximately meridional flow, which is northward in the upper and southward in the deep ocean. The cross-EJ structure of the vorticity-type flux components is summarised in Tables 1 and 2. Overall, across the EJ, the thickness flux works against the time-mean absolute PV gradient (appendix C), therefore it can not be approximated in terms of the diffusion process (e.g., as in Gent and McWilliams (1990)). Finally, with the sign reversal, the eddy flux patterns are qualitatively similar in the subtropical and subpolar gyres. This suggests that the results are robust with respect to $R e$, because the subpolar gyre is locally characterised by lower Re.

\subsection{Scale Interaction Components}

The pattern of the resolved eddy flux, $\left\langle\mathbf{F}^{\prime}{ }_{L L}\right\rangle$, is similar to that of $\left\langle\mathbf{F}^{\prime}\right\rangle$, but its divergence is different: it does not have the upper-ocean dipole pattern supporting the EJ (Fig. 6a). In the deep ocean it is largely opposite to the $F^{\prime}$-divergence, hence, it enhances the EJ — this is a result of the baroclinic instability that makes the jet more barotropic. Fluxes that are nonlocal in terms of the large-scale feedback on the eddies, $\left\langle\mathbf{F}^{\prime}{ }_{L E}\right\rangle$ and $\left\langle\mathbf{F}_{E L}^{\prime}\right\rangle$, tend to have opposite signs, but do not cancel each other completely, and their divergences have qualitative differences (Fig. 6b,c). The opposite sign is a result of mutual advection of the eddies and large-scale fluctuations. Around the EJ, the upper-ocean nonlocal fluxes are dominated by the stretching term: $\left\langle\mathbf{B}^{\prime}{ }_{L E}\right\rangle$ is meridionally down-gradient, and $\left\langle\mathbf{B}_{E L}^{\prime}\right\rangle$ is up-gradient. The 
EJ enhancement is due to the divergence of the zonal component of $\left\langle\mathbf{B}_{L E}^{\prime}\right\rangle$, which connects the western-boundary and EJ regions (Fig. 5). Here, the large-scale flow underestimates the transport of positive, boundary-generated PV to the north of the EJ, and it overestimates this transport to the south of it. The nonlocal eddy fluxes simply compensate for that. The $\left\langle\mathbf{F}_{E E}^{\prime}\right\rangle$ pattern that enhances the EJ is due to the sub-mesoscale eddy force variance, which is lost as a result of projecting the ER solution on the coarse grid. By the same mechanism, the $F^{\prime}$-variance drives the large-scale rectification (Berloff 2004).

\subsection{Dynamic Response to the Eddy Forcing}

What are dynamical contributions of different eddy forcing components? This question is answered by calculating the corresponding non-ER solutions. The wind forcing is neglected, and the solutions are obtained by running the eddy forcing history twice: for the spin-up and for the actual solution. It is found that both the time-mean, $\langle f\rangle$, and fluctuation, $\{f\}$, parts of the eddy forcing drive large-scale time-mean flows (Fig. 7a, b). The upper-ocean $\{f\}$-flow is characterised by the pair of broad and counter-rotating recirculations enhancing the EJ, and the shape of this pattern is strongly influenced by the spatial inhomogeneity of the eddy forcing variance (Berloff 2004). When the flow is decomposed into the time mean and fluctuations, it is found that the the large-scale flow pattern is due to the divergence of the resolved, up-gradient eddy PV flux around the EJ extension. This behaviour is consistent with the flow rectification mechanism studied in Haidvogel and Rhines (1983). The upper-ocean, time-mean $\langle f\rangle$-flow is characterised by both large-scale recirculations around the EJ and strong standing eddies that force the jet to meander. The standing eddies are largely driven by the relative vorticity fluxes (section 3.2). Also, $\langle f\rangle$-flow is characterised by cyclonic cells in the south-western corner of the basin. The EJ axes of the $\{f\}$ - and $\langle f\rangle$-solutions are substantially 
shifted to the south and north, respectively, of the full-force solution EJ. This is because the latitude of the maximum eddy forcing variance and the average latitude over which $\langle f\rangle$ changes sign do not coincide. The full-force solution incorporates all of the qualitative features of the $\{f\}$ - and $\langle f\rangle$-solutions (Fig. 7c), but it is not equal to their sum because of the nonlinear interactions. Also, this solution indicates that the time-mean and fluctuation eddy forcing partially compensate for each other. These results can be interpreted in a different way: the difference between the $f$ - and $\langle f\rangle$-solutions is the error associated with the absence of the eddy forcing fluctuations (Fig. 8a); and the difference between the $f$ - and $\{f\}$-solutions is the error due to the absence of the time-mean eddy forcing (Fig. 8b). These figures more explicitly show that both components enhance the EJ and the eddy forcing fluctuations are responsible for wiping out EJ recirculations induced by the time-mean eddy forcing.

Dynamical meaning of the eddy forcing is further investigated by calculating non-ER solutions driven by the vorticity-type eddy flux components: $\{B\},\langle B\rangle,\{R\}$, and $\langle R\rangle$ (Fig. 7d-i). The EJ is enhanced by all of the components except for the last one. The $\{R\}$-flow is stronger and its southern recirculation extends all the way to the southern boundary. The shape of the flow is largely set by the distribution of the $\{R\}$-variance, which has sharp maximum along the EJ. The variance is due to the relative vorticity supplied by the western boundary that is not converted into the stretching vorticity but is advected into the EJ region. The $\{B\}$-solution is characterised by weak anticyclonic recirculation. This asymmetry is due to the broad and relatively flat distribution of the $\{B\}$-variance, which facilitates partial compensation of the induced flow disturbances. (Some weakening can be also related to the details of the spacetime correlations of the $\{B\}$-force.) In the deep ocean, pattern of the $\{R\}$-solution is largely opposite to the upper-ocean one, because the deep-ocean boundary sources of relative vorticity 
are weak, and the dynamics is dominated by homogenisation of the absolute PV rather than by eddy forcing. The deep-ocean $\{B\}$-solution is characterised by several mesoscale recirculations, with the strongest cyclonic/anticyclonic pair that enhances the EJ. This behaviour occurs, perhaps, because thickness fluxes involve substantial vertical interactions, hence the deep ocean is more influenced by the strong, upper-ocean boundary source of PV.

The $\langle B\rangle$ - and $\langle R\rangle$-solutions indicate, in the agreement with results in section 3.1, that the EJ is largely maintained by the thickness flux. On the other hand, the standing-eddy pattern is due to the combined action of the $\langle B\rangle$ - and $\langle R\rangle$-fluxes. Finally, solutions driven by the combined vorticity-type eddy forcing are characterised by the same degree of mutual compensation as the full-force solution driven by the time-mean and fluctuation components.

\section{Summary}

The large-scale effects of the mesoscale eddies are studied in an eddy-resolving model of the midlatitude oceanic gyres. The new diagnostic method is proposed, which is based on dynamical decomposition of the flow into the large-scale and eddy components. The method yields the time history of the eddy forcing, which can be used as additional, external forcing in the corresponding non-eddy-resolving model of the gyres. The main strength of this approach is in its dynamical consistency: the non-eddy-resolving solution driven by the eddy forcing history correctly approximates the original large-scale flow component. It is shown that statistical decompositions, which are based on space-time filtering diagnostics, are dynamically inconsistent. The practical significance of the approach is in the idea that the dynamically consistent eddy forcing can be modelled as a random process (Berloff 2004). The new diagnostic-method algorithm is formulated and tested, and the diagnosed eddies, eddy fluxes, and eddy forcing 
are statistically and dynamically analysed.

It is argued that the main dynamic role of the eddies is to maintain the eastward-jet (EJ) extension of the subtropical western boundary current (WBC). This is done largely by the time-mean isopycnal-thickness flux and the relative-vorticity eddy flux fluctuations. Across the EJ, the thickness flux works against the time-mean absolute PV gradient, therefore it can not be approximated as the diffusion process, as suggested by Gent and McWilliams (1990). The time-mean meanders of the EJ and the structure of the WBC are maintained by the non-trivial, combined action of the relative-vorticity and thickness fluxes, however, there is a great deal of mutual compensation. The primary mechanism driving the thickness flux is baroclinic instability of the WBC that converts the boundary-generated relative vorticity into the stretching term. This flux connects the western-boundary and EJ regions and forms the large-scale gyre, which is the main instrument of the PV exchange between the subtropical gyre and the western boundary. The spatial nonlocality of this mechanism argues against local parameter closures that could relate the eddy forcing to the large-scale flow. Eddy fluxes driven by both the eddies and the large-scale flow are found to be important. The latter is typically neglected in the analysis, but here it corresponds to important large-scale feedback on the eddies. It is found that eddy flux fluctuations, which are also routinely neglected, drive very substantial large-scale response due to the nonlinear rectification process. Finally, two common theoretical relationships between the eddy effects and the large-scale flow are tested and are not confirmed.

The following developments of the results of this paper are anticipated. The dynamical decomposition method has to be extended for more realistic flows, and the low-frequency aspects and sub-mesoscale components of the eddy fluxes have to be studied as well. More 
optimal ways of coarse gridding and dynamic reduction as well as nonlocal parameter closures have to be looked for. Finally, based on the eddy flux diagnostics from the models, efficient strategies can be formulated for observations of the mesoscale eddies.

\section{Acknowledgements:}

Funding for this research was provided by NSF grant OCE 00-91836, by the Royal Society Fellowship, and by WHOI grants 27100056 and 52990035. The manuscript is WHOI contribution \#10931. 


\section{Appendix A}

\section{Parameters and Solution of the ER Model}

Parameters of the ER model are the following. The flow is driven at the surface by the asymmetric, double-gyre, zonal wind stress:

$$
\tau^{x}(y)=\tau_{0}\left[\cos \left(\frac{2 \pi(y-L / 2)}{L}\right)+2 \sin \left(\frac{\pi(y-L / 2)}{L}\right)\right]
$$

where $\tau_{0}=0.04 \mathrm{Nm}^{-2}$ and $L=3840 \mathrm{~km}$ is the size of the square basin (with $0 \leq y \leq L$, $0 \leq x \leq L)$. The lateral viscosity, that is, the eddy diffusivity, is $\nu=100 \mathrm{~m}^{2} \mathrm{~s}^{-1}$. The ocean is discretized vertically in 3 isopycnal layers with depths $H_{1}=200 \mathrm{~m}, H_{2}=1200 \mathrm{~m}$, and $H_{3}=2600$ m. The ratio of the density jumps across the layer interfaces is $\gamma=\left(\rho_{2}-\rho_{1}\right) /\left(\rho_{3}-\rho_{2}\right)=2$, which yields the first, $R d_{1}$, and second, $R d_{2}$, Rossby deformation radii of 52 and $30 \mathrm{~km}$. The stratification parameters in (2) are:

$$
\begin{array}{ll}
S_{i, 1}=f_{0}^{2}\left(H_{i} g \frac{\left(\rho_{i}-\rho_{i-1}\right)}{\rho_{1}}\right)^{-1}, & 1<i \leq N, \\
S_{i, 2}=f_{0}^{2}\left(H_{i} g \frac{\left(\rho_{i+1}-\rho_{i}\right)}{\rho_{1}}\right)^{-1}, & 1 \leq i<N,
\end{array}
$$

where $f_{0}=0.83 \times 10^{-4} \mathrm{~s}^{-1}$ is the mid-basin Coriolis parameter.

The ER model operates at large Reynolds number,

$$
R e=\frac{U L}{\nu}=\frac{\tau_{0}}{\rho_{1} H_{1} \beta \nu} \approx 1000
$$

where $U=\tau_{0}\left(\rho_{1} H_{1} L \beta\right)^{-1}$ is the upper-ocean Sverdrup velocity scale $\left(\approx 2.5 \mathrm{~cm} \mathrm{~s}^{-1}\right)$, and $\beta=2 \times 10^{-11} \mathrm{~m}^{-1} \mathrm{~s}^{-1}$. The horizontal grid resolution is uniform, with $513 \times 513$ grid points and $7.5-\mathrm{km}$ intervals between them, so that the Munk length scale, $\delta_{M}=(\nu / \beta)^{1 / 3}$, and the inertial length scale, $\delta_{I}=(U / \beta)^{1 / 2}$, are resolved by more than 2 grid points. The first and second Rossby radii are resolved by 7 and 4 grid points, respectively. This grid size is close to 
the 1/12-degree resolution, at which, it is argued, the mesoscale eddies are marginally resolved (e.g., Bleck et al. 1995). The QG equations (1) and (2) are discretized with the second-order finite differences, including Arakawa scheme, and time stepped with the leapfrog scheme and 0.6 hours time step.

The upper-ocean time-mean circulation (Fig. 1a) consists of the southern (subtropical) and northern (subpolar) gyres that fill about $2 / 3$ and $1 / 3$ of the basin, respectively, which is consistent with the wind stress pattern. The time-mean flow is characterised by the Sverdrup balance in the eastern part of the basin, and by the pair of the WBCs and their EJ extensions in the western part of the basin — this is a robust regime that appears with the no-slip boundary condition and at large $R e$ (Haidvogel et al. 1992). In terms of the fluctuations, the basin can be partitioned into more energetic "western" part, characterised by strong vortices, and less energetic "eastern" part, dominated by the planetary waves (see Berloff et al. 2002 for the details). In the deep ocean, the eddies are generally weaker, but they drive time-mean flow in the western basin (Fig. 1c,d). 


\section{Appendix B}

\section{Statistical Decomposition of the Flow: Examples}

Statistical decomposition of turbulent flows into the large-scale and eddy components is a common approach, and in the ocean dynamics, the most common choice of a statistical filter (10) is the infinite-time averaging. For each choice of the filter, which is not obviously constrained by physical arguments, there is unique eddy flux history. However, dynamical meaning of such eddy flux is unclear: when its history is used as the external forcing for an arbitrary non-ER model, there is no guarantee that the resulting non-ER solution will approximate the original flow, as it has to. Dynamically consistent eddy fluxes has no such problem. Below, the failure with dynamical interpretation of the common statistical approach is illustrated with two cases, both of which yield non-ER solutions of poor quality: the case A involves heavy filtering in time only; and $\mathbf{B}$ involves moderate filtering in space only. A similar approach, which is based on calculating the eddy forcing with respect to the flow that is moderately filtered in time, is considered by Marshall and Molteni (1993), however they correct the dynamic model by the time-mean component rather than the full history of the eddy forcing.

In $\mathbf{A}$, the symmetric, 100-day moving-window averaging is used - this decomposition is qualitatively similar to the common infinite-time averaging. The non-ER solution forced by the $\mathbf{A}$-decomposed eddy forcing has largely incorrect time-mean and instantaneous flows, and the time-mean eddy flux divergence (Fig. 9a,c). The global potential and kinetic energy time series of the non-ER circulation have no significant correlation with the ER time series, and the time-mean kinetic and potential energies are 5 and 1.5 times larger than the corresponding ER energies. In B, 7 iterations of the gridscale, 9-point Gaussian filter are used, so that 
the resulting large-scale streamfunction resembles the dynamically filtered one. The non-ER solution forced by the corresponding eddy forcing history is more accurate (compare Fig. 9b,d with Figs. 1c and 4f), but there are still significant errors. In particular, recirculations around the EJ are overestimated and the potential and kinetic energy time series have rather poor correlations with the ER values: 0.42 and 0.25 , respectively. Overall, the relative success of the solution B suggests that the optimal, spatially inhomogeneous and stationary, statistical filter (10) may exist, but it is not clear how to find it. 


\section{Appendix $\mathrm{C}$}

\section{Relationships Between the Eddy Fluxes and the Background Flow}

Here, two parameter closures for the eddies are tested, and none of them is confirmed. Hypothetically, the time-mean eddy flux of absolute PV (or, PV anomaly) can be linearly related to the local gradient of the time-mean absolute PV:

$$
\left\langle\mathbf{F}^{\prime}\right\rangle=-\mathbf{K} \nabla\langle\bar{Q}\rangle
$$

where $\mathbf{K}$ is the corresponding eddy diffusivity (tensor) coefficient. With (C1), the eddy forcing can be related to the large-scale flow:

$$
f^{\prime}=-\nabla \cdot\left\langle\mathbf{F}^{\prime}\right\rangle=\nabla \cdot \mathbf{K} \nabla\langle\bar{Q}\rangle
$$

This relationship can be put into practice if $\mathbf{K}$ is positive definite, and, therefore, the eddy flux is down-gradient, but this is not always the case in the geophysical turbulence (e.g., Starr 1968). Even in the down-gradient case, finding relationships between $\mathbf{K}$ and the large-scale flow is problematic.

Relationships between the dynamically consistent eddy flux and the time-mean, large-scale flow are summarised in Tables 1 and 2. At all depths, $\langle\bar{q}\rangle$ is positive in the near-shore side of the subtropical WBC, because of the relative vorticity component, but elsewhere it is dominated by the stretching term, therefore, it changes sign from the upper to middle layers. In the EJ region, the change of sign results in: $\partial\langle\bar{q}\rangle / \partial y>0$ in the upper, and $\partial\langle\bar{q}\rangle / \partial y<0$ in the deep ocean. The cross-EJ gradient of $\langle\bar{Q}\rangle$ is positive at all depths, but it is larger in the upper

ocean. Given that, the upper-ocean cross-EJ flux of $\langle\bar{Q}\rangle$ is up-gradient. (Here, the planetary vorticity contribution to the eddy flux is negligible, because $\left\langle v^{\prime}\right\rangle \approx 0$.) On the other hand, the deep-ocean cross-EJ flux is down-gradient, which is consistent with the idea of local PV 
homogenisation by the eddies. Thus, both the up- and down-gradient eddy fluxes are found, and the most important dynamically, upper-ocean, cross-EJ flux is up-gradient, which is a strong argument against the diffusion hypothesis. Finally, the up-gradient eddy fluxes in the EJ region are not found in the single-gyre barotropic solutions at similar Re (Fox-Kemper and Pedlosky 2004), which is consistent with the crucial role of the thickness fluxes.

Is it possible to relate the eddy forcing to the intensity of the eddies? The statistical similarity hypothesis (DelSole 2001) states that local rate of the eddy enstrophy production by the eddy forcing is linearly proportional to local enstrophy:

$$
\left\langle q_{i}^{\prime} f_{i}\right\rangle(\mathbf{x})=C_{S I M}\left\langle q_{i}^{\prime 2}\right\rangle(\mathbf{x}),
$$

where $C_{S I M}$ is the timescale parameter. The enstrophy distribution is a large-scale quantity, which can be potentially related to the large-scale flow. Here, (C3) is tested directly from the eddy diagnostics, by plotting scatterplot of both sides of the relationship against each other. The resulting broad scatter disproves the hypothesis. The approximate linear fit yields $C_{S I M}$ of about 115 and 193 days in the upper and deep ocean, respectively, which indicates that the upper-ocean eddy forcing is more efficient in generating enstrophy. Overall, the statistical similarity hypothesis is unlikely to work in situations when strong eddies yield mutually compensated nonlinear interactions, which is the case here, with coherent circular vortices and finite-amplitude Rossby waves. 


\section{References}

Berloff, P., 2004: Random-forcing model of the mesoscale oceanic eddies. J. Fluid Mech., in press.

Berloff, P., J. McWilliams, and A. Bracco, 2002: Material transport in oceanic gyres. Part I: Phenomenology. J. Phys. Oceanogr. 32, 764-796.

Berloff, P., and J. McWilliams, 1999a: Large-scale, low-frequency variability in wind-driven ocean gyres. J. Phys. Oceanogr., 29, 1925-1949.

Berloff, P., and J. McWilliams, 1999b: Quasigeostrophic dynamics of the western boundary current. J. Phys. Oceanogr., 29, 2607-2634.

Berloff, P., and S. Meacham, 1998: On the stability of the wind-driven circulation. J. Mar. Res., 56, 937-993.

Bleck, R., S. Dean, M. O'Keefe, and A. Sawdey, 1995: A comparison of data-parallel and messagepassing versions of the Miami Isopycnic Coordinate Ocean Model (MICOM). Parallel Computing, 1695-1720.

Bryan K., 1963: A numerical investigation of a nonlinear model of a wind-driven ocean. J. Atmos. Sc., 20, 594-606

Bryan F., 1987: Parameter sensitivity of primitive equation ocean general circulation model. J. Phys. Oceanogr., 17, 970-985.

Cessi, P., 1991: Laminar separation of colliding western boundary currents. J. Mar. Res., 49, 697-717.

Cessi, P., and G. Ierley, 1995: Symmetry-breaking multiple equilibria in quasi-geostrophic winddriven flows. J. Phys. Oceanogr., 25, 1196-1205.

Cessi, P., G. Ierley, and W. Young, 1987: A model of the inertial recirculation driven by potential vorticity anomalies. J. Phys. Oceanogr., 17, 1640-1652. 
D'Andrea, F., and R. Vautard, 2000: Reducing systematic errors by empirically correcting model errors. Tellus, 52A, 21-41.

DelSole, T., 2001: A theory for the forcing and dissipation in stochastic turbulence models. $J$. Atmos. Sci., 58, 3762-3775.

Domaradzki, J., K. Loh, and P. Yee, 2002: Large eddy simulations using the subgrid-scale estimation model and truncated Navier-Stokes dynamics. Theoret. Comput. Fluid Dynamics, 15, 421450.

Drijfhout, S., and W. Hazeleger, 2001: Eddy mixing of potential vorticity versus thickness in an isopycnic ocean model. J. Phys. Oceanogr., 31, 481-505.

Fofonoff, N., 1954: Steady flow in a frictionless homogeneous ocean. J. Marine Res., 13, 254-262.

Fox-Kemper, B., R. Ferrari, and J. Pedlosky, 2003: On the indeterminacy of rotational and divergent eddy fluxes. J. Phys. Oceanogr., 33, 478-483.

Fox-Kemper, B., and J. Pedlosky, 2004: Wind-driven barotropic gyre I: Circulation control by eddy vorticity fluxes to an enhanced removal region. J. Mar. Res., 62, 169-193.

Gent, P., and J. McWilliams, 1990: Isopycnal mixing in ocean circulation models. J. Phys. Oceanogr., 20, $150-155$.

Haidvogel, D., J. McWilliams, and P. Gent, 1992: Boundary current separation in a quasigeostrophic, eddy-resolving ocean circulation model. J. Phys. Oceanogr., 22, 882-902.

Haidvogel, D., and P. Rhines, 1983: Waves and circulation driven by oscillatory winds in an idealized ocean basin. Geophys. Astrophys. Fluid Dynamics, 25, 1-63.

Harrison, D., 1981: Eddy lateral vorticity transport and the equilibrium of the North Atlantic subtropical gyre. J. Phys. Oceanogr., 11, 1154-1158.

Hogg, N., 1993: Toward parameterization of the eddy field near the Gulf Stream. Deep-Sea Res., 
40, 2359-2376.

Holland, W., 1978: The role of mesoscale eddies in the general circulation of the ocean - Numerical experiments using a wind-driven quasigeostrophic model. J. Phys. Oceanogr., 8, 363-392.

Jayne, S., N. Hogg, and P. Malanotte-Rizzoli, 1996: Recirculation gyres forced by a beta-plane jet. J. Phys. Oceanogr., 26, 492-504.

Jayne, S., and N. Hogg, 1999: On recirculation forced by an unstable jet. J. Phys. Oceanogr., 29, $2711-2718$.

Kaas, E., A. Guldberg, W. May, and M. Deque, 1999: Using tendency errors to tune parameterization of unresolved dynamical scale interactions in atmospheric general circulation models. Tellus, 51A, 612-629.

Marshall, J., 1984: Eddy-mean-flow interaction in a barotropic ocean model. Quat. J. Royal Met. Soc., 110, 573-590.

Marshall, J., and F. Molteni, 1993: Toward a dynamical understanding of planetary-scale flow regimes. J. Atmos. Sci., 50, 1792-1818.

McIntyre, M., 2000: On global-scale atmospheric circulations. In: Perspectives in Fluid Dynamics: A Collective Introduction to Current Research, ed. G. Batchelor, H. Moffat, and M. Worster, 557-624. Cambridge, University Press, 631 pp.

Moore, D., 1963: Rossby waves in ocean circulation. Deep-Sea Res., 10, 735-748.

Munk, W., 1950: On the wind-driven ocean circulation. J. Meteorol., 7, 79-93.

Pedlosky, J., 1987: Geophysical Fluid Dynamics. 2nd edn., Springer-Verlag, 710 pp.

Roberts, M., and D. Marshall, 2000: On the validity of downgradient eddy closures in ocean models. J. Geophys. Res., 105, 28613-28627.

Sheremet, V., G. Ierley, and V. Kamenkovich, 1997: Eigenanalysis of the two-dimensional wind- 
driven ocean circulation problem. J. Mar. Res., 55, 57-92.

Siegel, A., J. Weiss, J. Toomre, J. McWilliams, P. Berloff, and I. Yavneh, 2001: Eddies and vortices in ocean basin dynamics. Geophys. Res. Lett., 28, 3183-3187.

Speich, S., H. Dijkstra, and M. Ghil, 1995: Successive bifurcations in a shallow-water model applied to the wind-driven ocean circulation. Nonlin. Proc. Geophys., 2, 241-268.

Starr, V., 1968: Physics of negative viscosity phenomena. McGraw-Hill, New York, 256 pp.

Stommel, H., 1948: The westward intensification of wind-driven oceanic currents. Trans. Am. Geophys. Union 29, 202-206.

Treguier, A., 1999: Evaluating eddy mixing coefficients from eddy-resolving ocean model: A case study. J. Mar. Res., 57, 89-108.

Veronis, G., 1966: Wind-driven ocean circulation. Part 2. Deep-Sea Res., 13, 31-55. 


\section{Figure Captions}

Fig. 1. ER (a, b) time-mean and (c, d) instantaneous, total velocity streamfunctions in the (a, c) upper and (b, d) deep ocean. Contour intervals (CI): (a, c) $10^{4} \mathrm{~m}^{2} \mathrm{~s}^{-1}$, (b) $0.25 \times 10^{4} \mathrm{~m}^{2} \mathrm{~s}^{-1}$, and (d) $0.5 \times 10^{4} \mathrm{~m}^{2} \mathrm{~s}^{-1}$.

Fig. 2. The CG, upper-ocean velocity streamfunction: (a, b) time-mean, and (c, d) instantaneous. The eddy viscosity, $\bar{\nu}$ is (a, c) 110 and (b, d) $1000 \mathrm{~m}^{2} \mathrm{~s}^{-1}$. CI $=10^{4} \mathrm{~m}^{2} \mathrm{~s}^{-1}$.

Fig. 3. Streamfunction of the upper-ocean non-ER solution: (a) $\left\langle\overline{\psi_{i}(x, y)}\right\rangle$, (b) $\overline{\psi_{i}(x, y)}$, and $(\mathrm{c}) \psi_{i}^{\prime}(x, y)\left(\mathrm{CI}=10^{4} \mathrm{~m}^{2} \mathrm{~s}^{-1}\right)$.

[Note to the technical editor: Please, put Figs. 1 and 3 on opposing pages for ready comparison.]

Fig. 4. The upper-ocean relative vorticity, $\left\langle\mathbf{R}^{\prime}\right\rangle$, (upper) and isopycnal thickness anomaly, $\left\langle\mathbf{B}^{\prime}\right\rangle$, (lower row of panels) components of the time-mean eddy flux and their horizontal divergences. The (a,d) zonal and (b, e) meridional flux components, and (c, f) the flux divergences. The corresponding upper-ocean velocity streamfunction, $\bar{\psi}$, is shown with $\mathrm{CI}=10^{4} \mathrm{~m}^{2} \mathrm{~s}^{-1}$. Small rectangular shown in panels (c) and (d) outlines the basin subdomain used in Fig. 5. The positive (negative) fluxes and divergences are nondimensionalised by their corresponding maximum (minimum) values, so that the color scale changes from -1 to 1 . This is routinely done in all the other color figures. Here, the minimum and maximum values of the color-plotted quantities (units for fluxes: $10^{-3} \mathrm{~m} \mathrm{~s}^{-2}$; for divergences: $10^{-8} \mathrm{~s}^{-2}$ ) are: (a, d) -39 and 51 , (b, e) -120 and $53,(\mathrm{c}, \mathrm{f})-42$ and 15.6 .

Fig. 5. Streamlines of the upper-ocean eddy fluxes of (a) relative vorticity, $\left\langle\mathbf{R}^{\prime}\right\rangle$, and (b) isopycnal thickness anomaly, $\left\langle\mathbf{B}^{\prime}\right\rangle$, are shown with black lines and arrows. The color shading 
indicates the flux divergences. The basin subdomain is outlined in Fig. 4, and one of the corresponding velocity streamfunction levels (thick green line) indicates, for convenience, location of the EJ. The minimum and maximum values of the divergence are -39 and $15.6 \times 10^{-8} \mathrm{~S}^{-2}$.

Fig. 6. Upper-ocean divergences of the time-mean scale interaction components: (a) $\left\langle\mathbf{F}_{L L}\right\rangle$, (b) $\mathbf{F}_{L E}^{\prime},(\mathrm{c})\left\langle\mathbf{F}_{E L}^{\prime}\right\rangle$, and (d) $\mathbf{F}_{E E}^{\prime}$. The upper-ocean, time-mean velocity streamfunction, $\langle\bar{\psi}\rangle$, is shown with $\mathrm{CI}=10^{4} \mathrm{~m}^{2} \mathrm{~s}^{-1}$. The minimum and maximum values of the color-plotted divergences (units: $10^{-8} \mathrm{~S}^{-2}$ ): (a) -25.0 and 79.9, (b) -61.9 and 19.7, (c) -25.4 and 33.7, and (d) -33.7 and 34.4 .

Fig. 7. The time-mean, upper-ocean velocity streamfunction of the non-ER solutions forced by the different components of the eddy forcing, and with no wind forcing. (a) $f=\{f\}$, (b) $f=\langle f\rangle$, (c) $f=\langle f\rangle+\{f\}$, (d) $\{B\}$, (e) $\langle B\rangle$, (f) $\{B\}+\langle B\rangle$, (g) $\{R\}$, (h) $\langle R\rangle$, and (i) $\{R\}+\langle R\rangle . \mathrm{CI}=0.25 \times 10^{4} \mathrm{~m}^{2} \mathrm{~s}^{-1}$ in $(\mathrm{a}, \mathrm{d}, \mathrm{g})$, and $\mathrm{CI}=0.5 \times 10^{4} \mathrm{~m}^{2} \mathrm{~s}^{-1}$ otherwise.

Fig. 8. Error due to the absence of the (a) fluctuation and (b) time-mean components of the eddy forcing $\left(\mathrm{CI}=0.5 \times 10^{4} \mathrm{~m}^{2} \mathrm{~s}^{-1}\right)$.

Fig. 9. The upper-ocean, time-mean velocity streamfunctions, $\langle\bar{\psi}\rangle,\left(\mathrm{CI}=10^{4} \mathrm{~m}^{2} \mathrm{~s}^{-1}\right)$ and the corresponding divergences of the time-mean eddy flux, $\left\langle\mathbf{F}^{\prime}\right\rangle$, (color) that are obtained with the statistical decompositions (a) A and (b) B (see appendix B). The corresponding streamfunction snapshots, which are analogous to those in Figs. 1c and 3b, are shown in panels (c) and (d), respectively. The minimum and maximum values of the color plotted quantities are: (a) -25.3 and $18.4 \times 10^{-8} \mathrm{~s}^{-2}$, (b) -23.5 and $8.7 \times 10^{-8} \mathrm{~S}^{-2}$. 


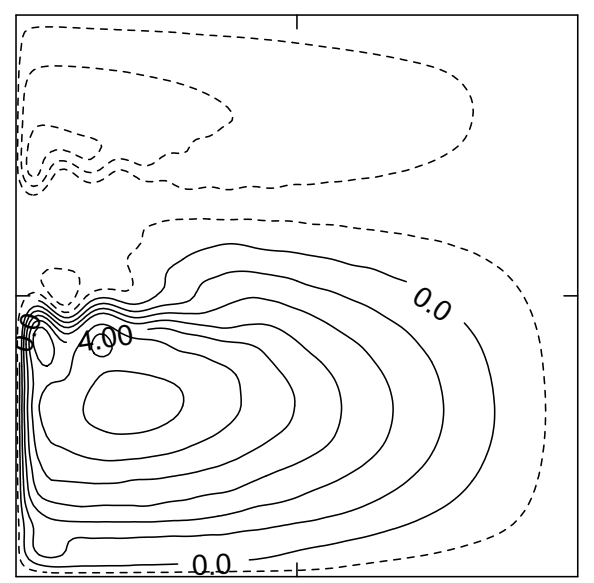

(a)

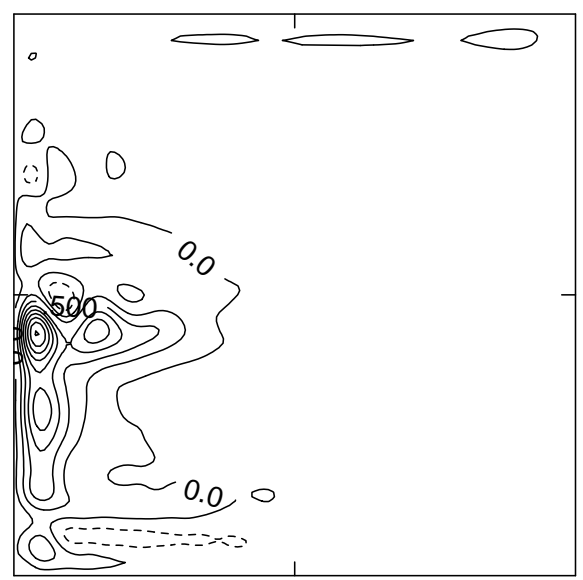

(b)

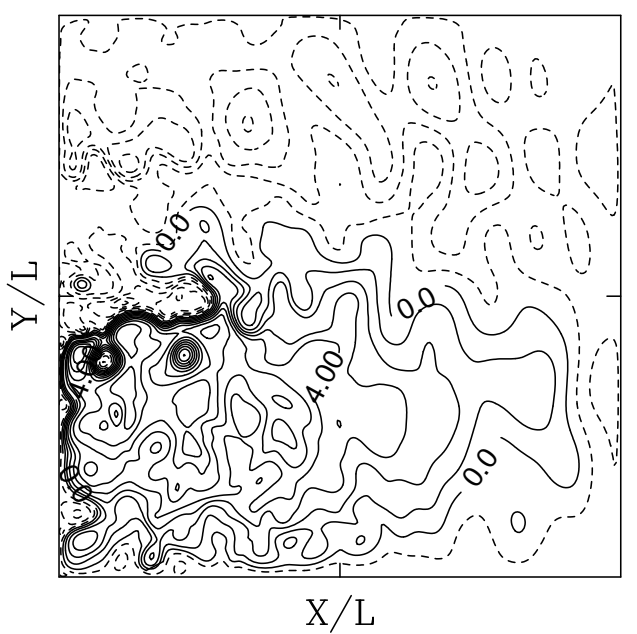

(c)

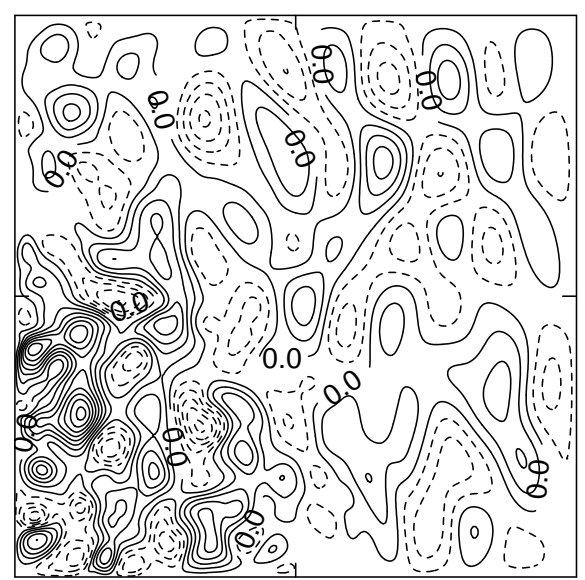

(d)

Figure 1: ER (a, b) time-mean and $(\mathrm{c}, \mathrm{d})$ instantaneous, total velocity streamfunctions in the (a, c) upper and (b, d) deep ocean. Contour intervals (CI): (a, c) $10^{4} \mathrm{~m}^{2} \mathrm{~s}^{-1}$, (b) $0.25 \times 10^{4} \mathrm{~m}^{2} \mathrm{~s}^{-1}$, and (d) $0.5 \times 10^{4} \mathrm{~m}^{2} \mathrm{~s}^{-1}$. 

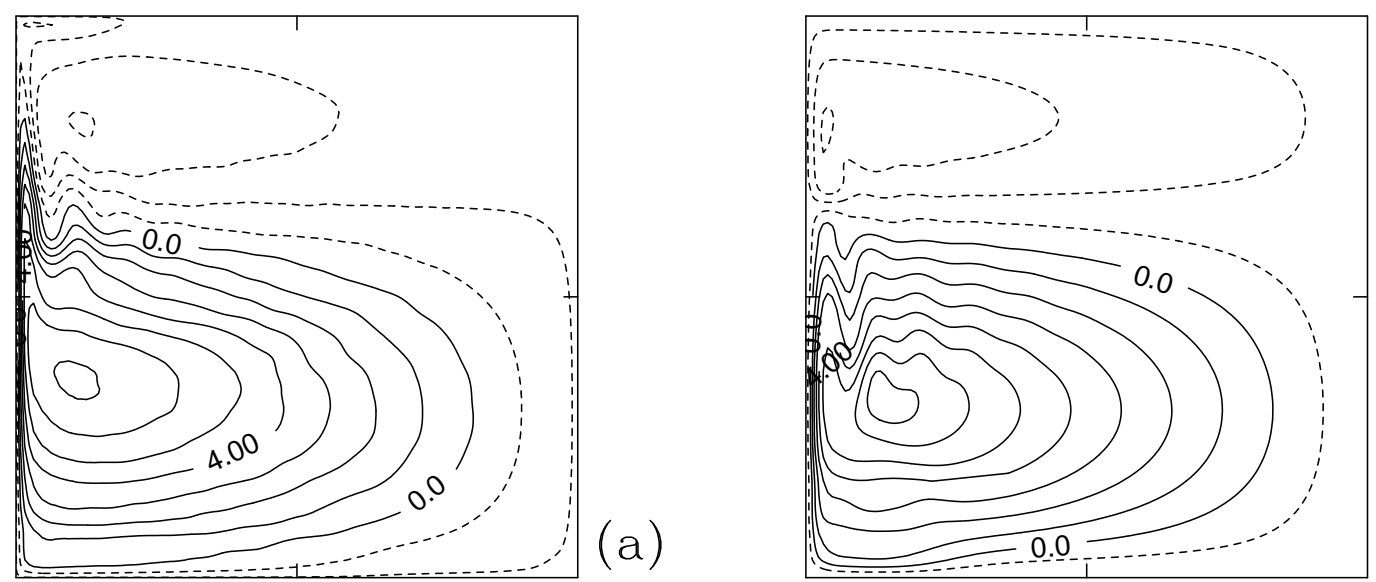

(b)

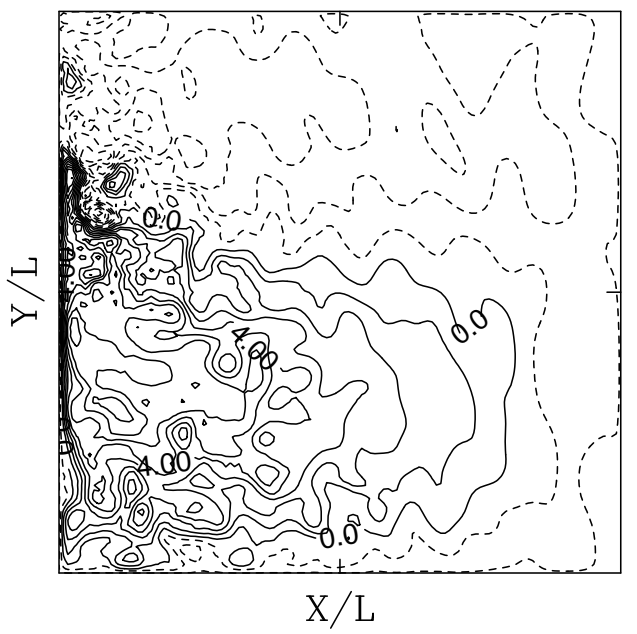

(c)

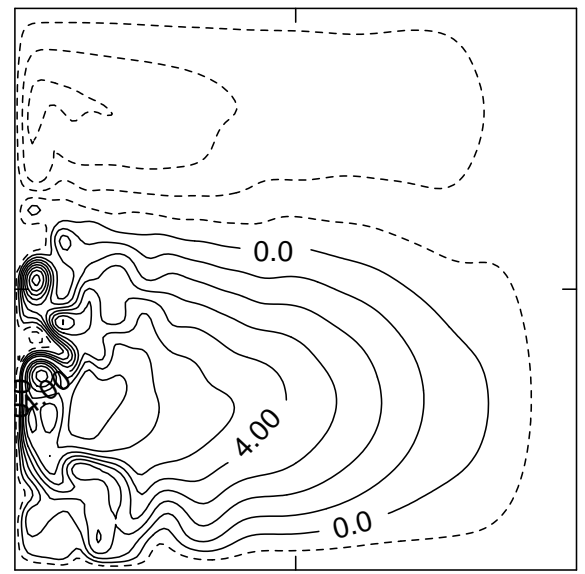

(d)

Figure 2: The CG, upper-ocean velocity streamfunction: (a, b) time-mean, and (c, d) instantaneous. The eddy viscosity, $\bar{\nu}$ is (a, c) 110 and (b, d) $1000 \mathrm{~m}^{2} \mathrm{~s}^{-1}$. CI $=10^{4} \mathrm{~m}^{2} \mathrm{~s}^{-1}$. 

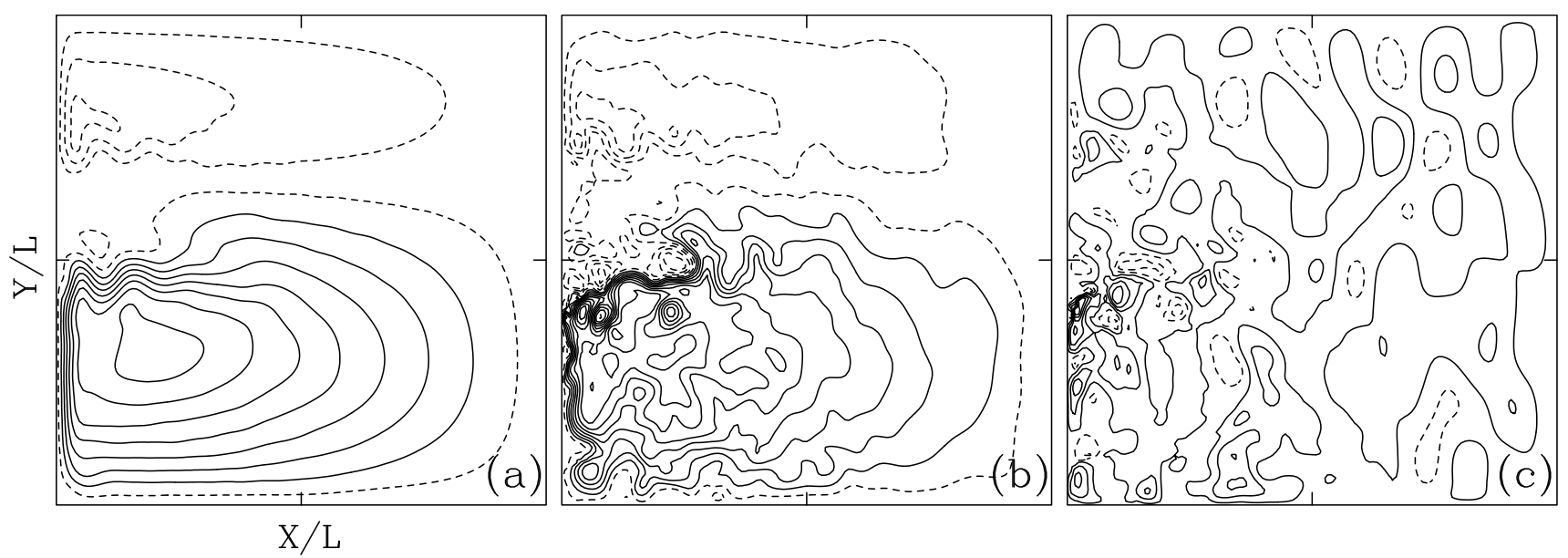

Figure 3: Streamfunction of the upper-ocean non-ER solution: (a) $\left\langle\overline{\psi_{i}(x, y)}\right\rangle$, (b) $\overline{\psi_{i}(x, y)}$, and (c) $\psi_{i}^{\prime}(x, y)\left(\mathrm{CI}=10^{4} \mathrm{~m}^{2} \mathrm{~s}^{-1}\right)$. 

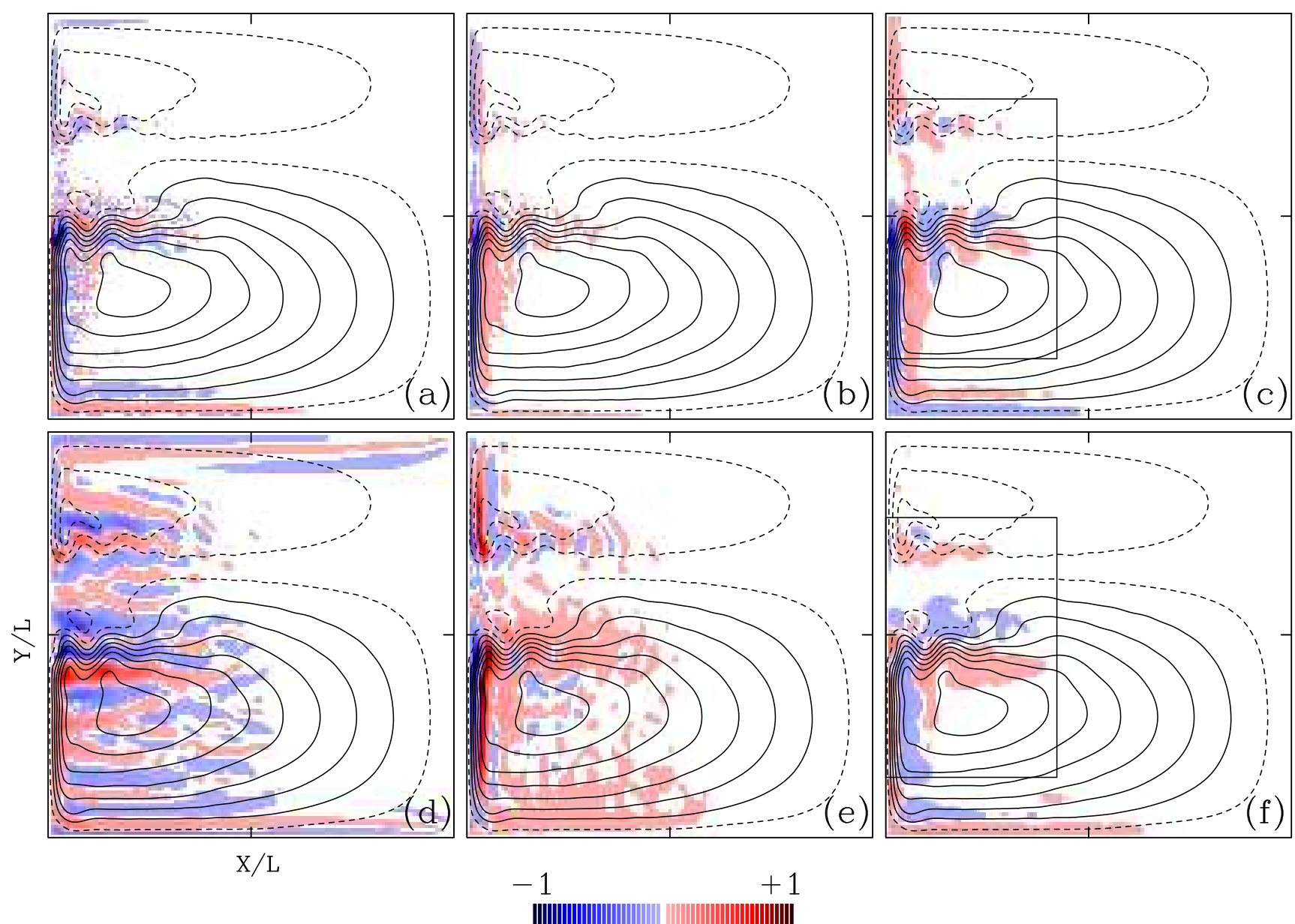

Figure 4: The upper-ocean relative vorticity, $\left\langle\mathbf{R}^{\prime}\right\rangle$, (upper) and isopycnal thickness anomaly, $\left\langle\mathbf{B}^{\prime}\right\rangle$, (lower row of panels) components of the time-mean eddy flux and their horizontal divergences. The (a,d) zonal and (b, e) meridional flux components, and (c, f) the flux divergences. The corresponding upper-ocean velocity streamfunction, $\bar{\psi}$, is shown with $\mathrm{CI}=10^{4} \mathrm{~m}^{2} \mathrm{~s}^{-1}$. Small rectangular shown in panels (c) and (d) outlines the basin subdomain used in Fig. 5. The positive (negative) fluxes and divergences are nondimensionalised by their corresponding maximum (minimum) values, so that the color scale changes from -1 to 1 . This is routinely done in all the other color figures. Here, the minimum and maximum values of the color-plotted quantities (units for fluxes: $10^{-3} \mathrm{~m} \mathrm{~s}^{-2}$; for divergences: $10^{-8} \mathrm{~s}^{-2}$ ) are: (a, d) -39 and 51 , (b, e) -120 and $53,(\mathrm{c}, \mathrm{f})-42$ and 15.6 . 

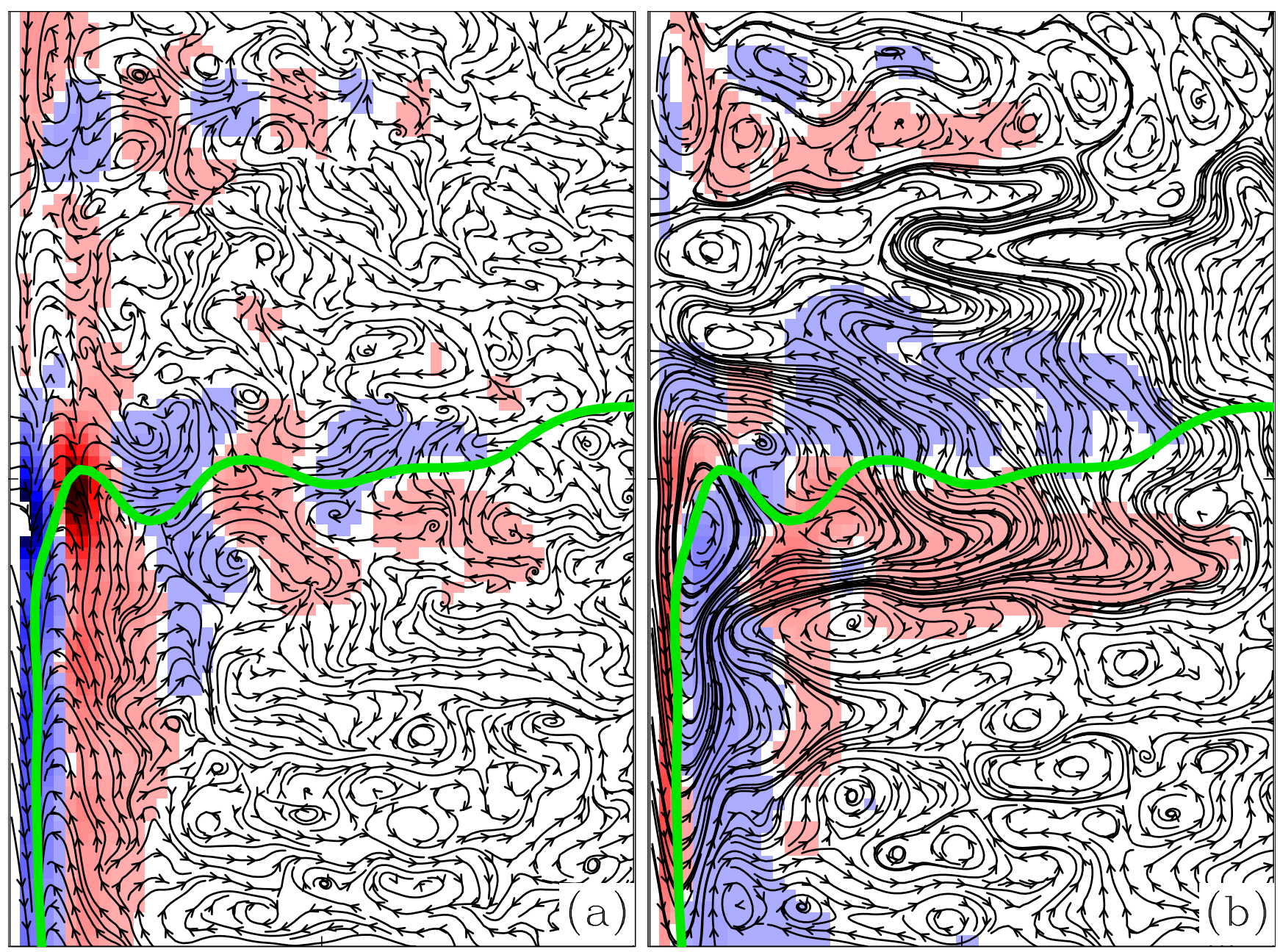

Figure 5: Streamlines of the upper-ocean eddy fluxes of (a) relative vorticity, $\left\langle\mathbf{R}^{\prime}\right\rangle$, and (b) isopycnal thickness anomaly, $\left\langle\mathbf{B}^{\prime}\right\rangle$, are shown with black lines and arrows. The color shading indicates the flux divergences. The basin subdomain is outlined in Fig. 4, and one of the corresponding velocity streamfunction levels (thick green line) indicates, for convenience, location of the EJ. The minimum and maximum values of the divergence are -39 and $15.6 \times 10^{-8} \mathrm{~s}^{-2}$. 

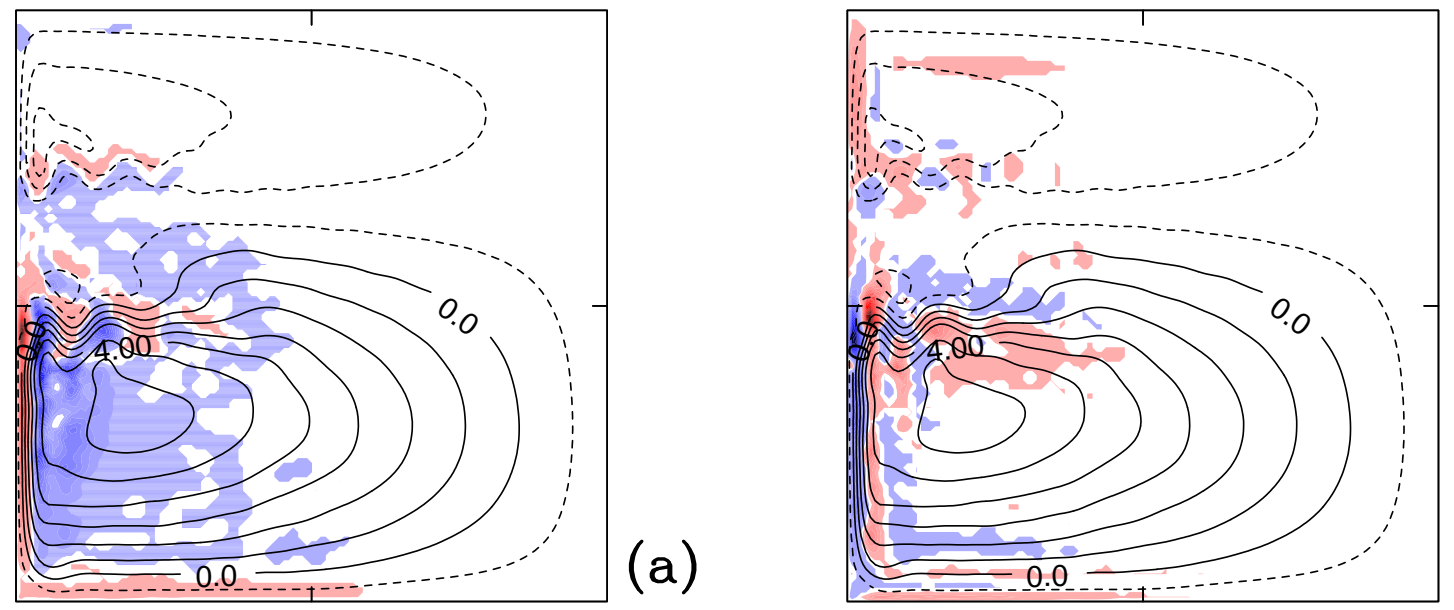

(b)

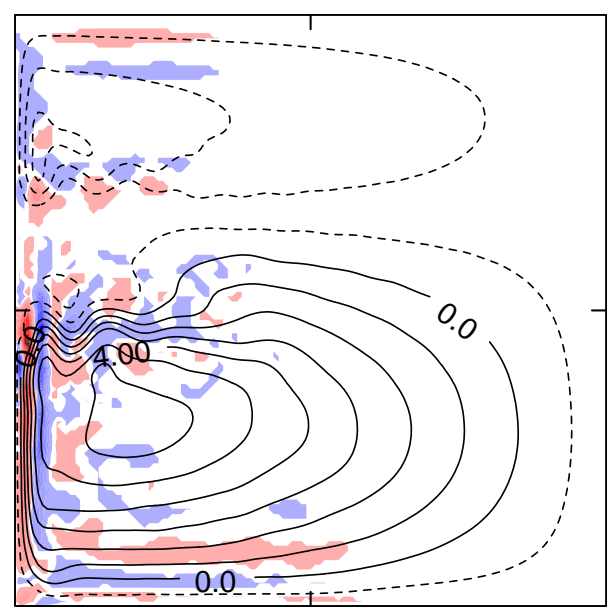

(c)

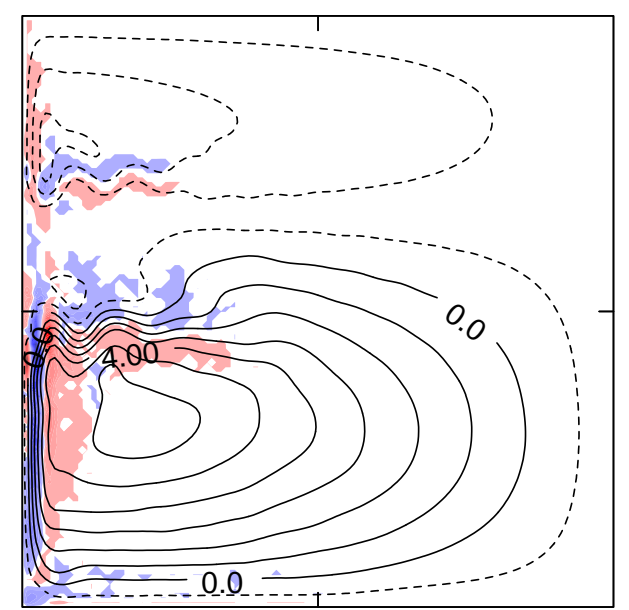

(d)

Figure 6: Upper-ocean divergences of the time-mean scale interaction components: (a) $\left\langle\mathbf{F}_{L L}\right\rangle$, (b) $\mathbf{F}_{L E}^{\prime}$, (c) $\left\langle\mathbf{F}_{E L}^{\prime}\right\rangle$, and (d) $\mathbf{F}_{E E}^{\prime}$. The upper-ocean, time-mean velocity streamfunction, $\langle\bar{\psi}\rangle$, is shown with $\mathrm{CI}=10^{4} \mathrm{~m}^{2} \mathrm{~s}^{-1}$. The minimum and maximum values of the color-plotted divergences (units: $10^{-8} \mathrm{~s}^{-2}$ ): (a) -25.0 and 79.9, (b) -61.9 and 19.7, (c) -25.4 and 33.7, and (d) -33.7 and 34.4 . 

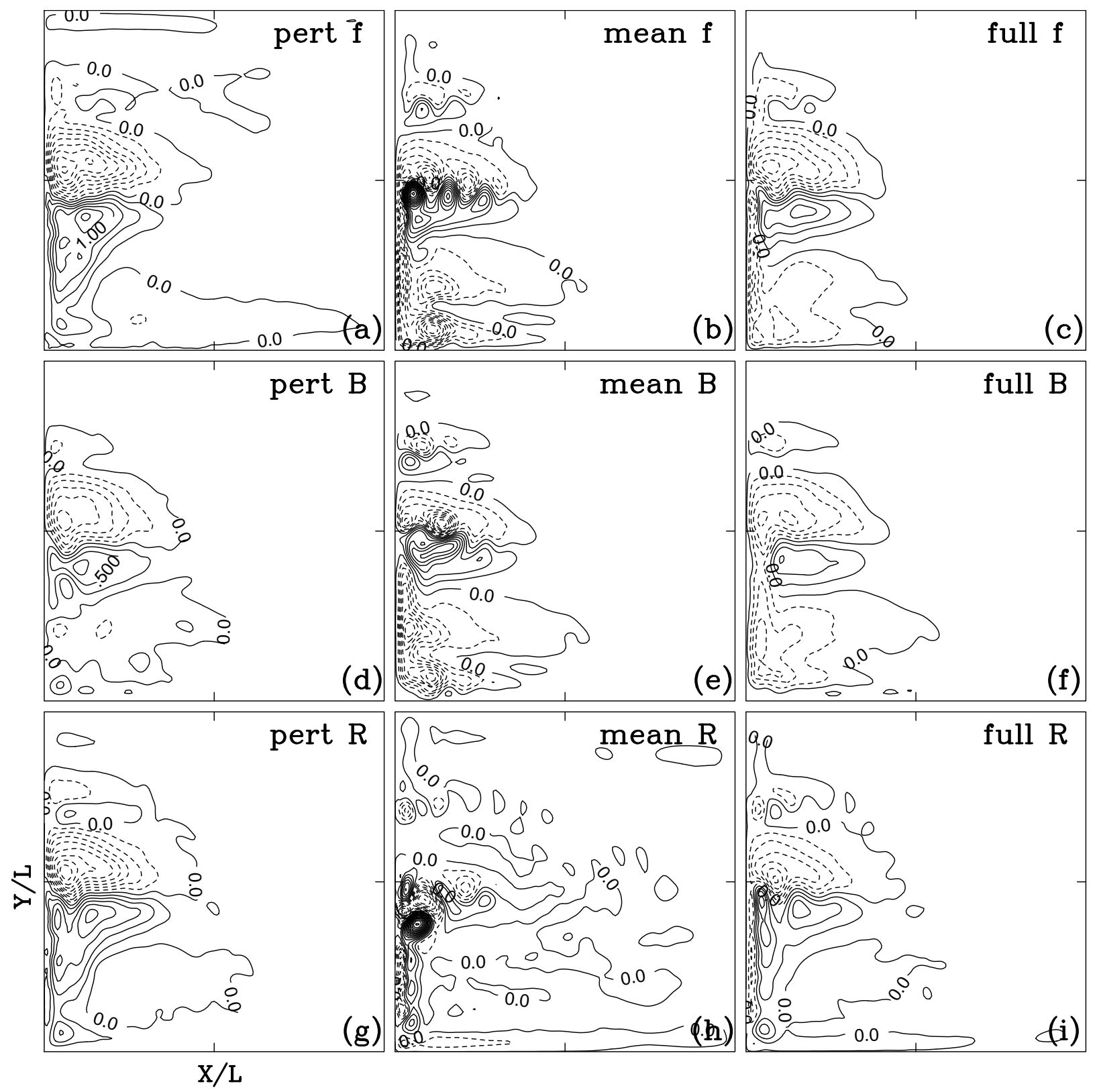

Figure 7: The time-mean, upper-ocean velocity streamfunction of the non-ER solutions forced by the different components of the eddy forcing, and with no wind forcing. (a) $f=\{f\}$, (b) $f=\langle f\rangle$, (c) $f=\langle f\rangle+\{f\}$, (d) $\{B\}$, (e) $\langle B\rangle$, (f) $\{B\}+\langle B\rangle$, (g) $\{R\}$, (h) $\langle R\rangle$, and (i) $\{R\}+\langle R\rangle . \mathrm{CI}=0.25 \times 10^{4} \mathrm{~m}^{2} \mathrm{~s}^{-1}$ in $(\mathrm{a}, \mathrm{d}, \mathrm{g})$, and $\mathrm{CI}=0.5 \times 10^{4} \mathrm{~m}^{2} \mathrm{~s}^{-1}$ otherwise. 

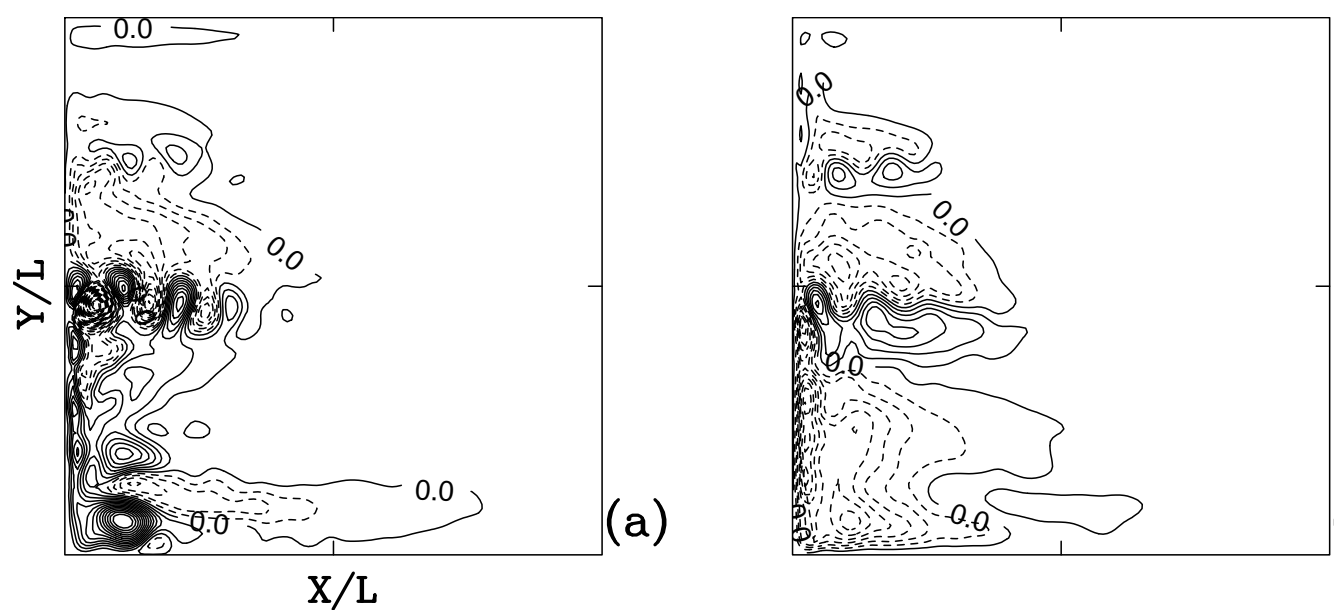

(b)

Figure 8: Error due to the absence of the (a) fluctuation and (b) time-mean components of the eddy forcing $\left(\mathrm{CI}=0.5 \times 10^{4} \mathrm{~m}^{2} \mathrm{~s}^{-1}\right)$. 

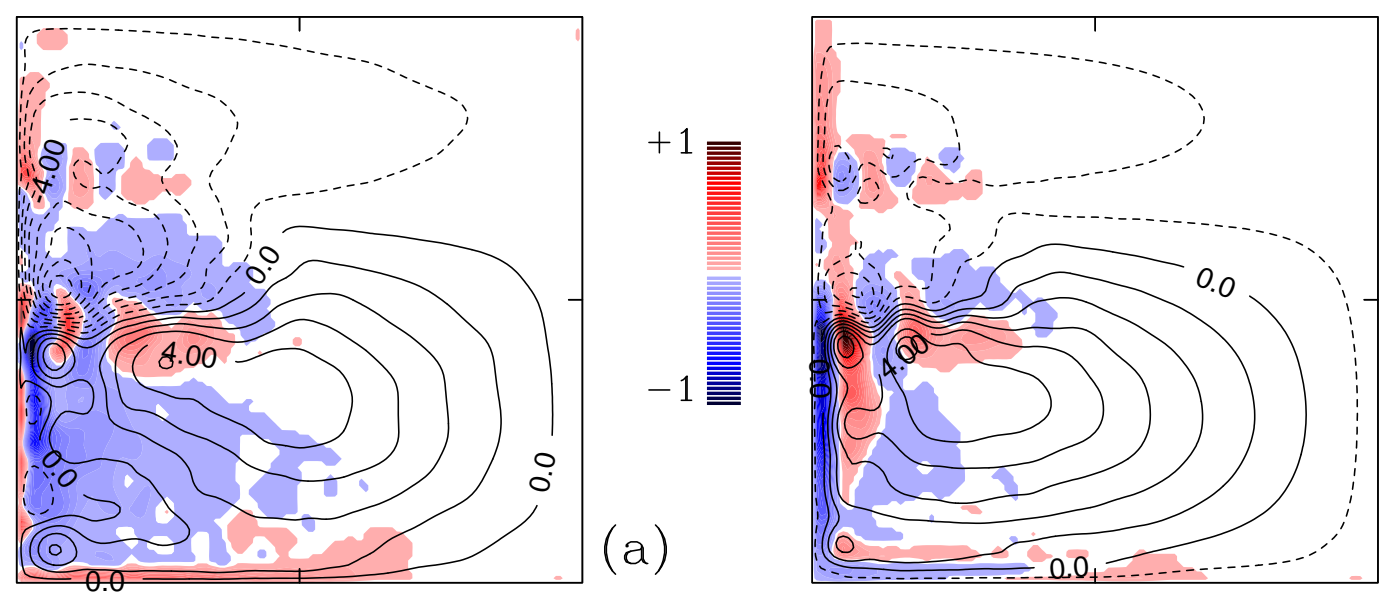

(b)

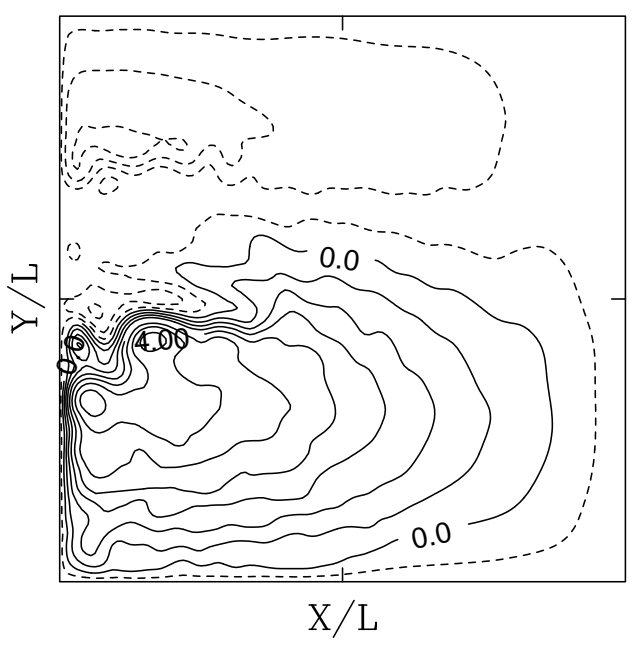

$(c)$

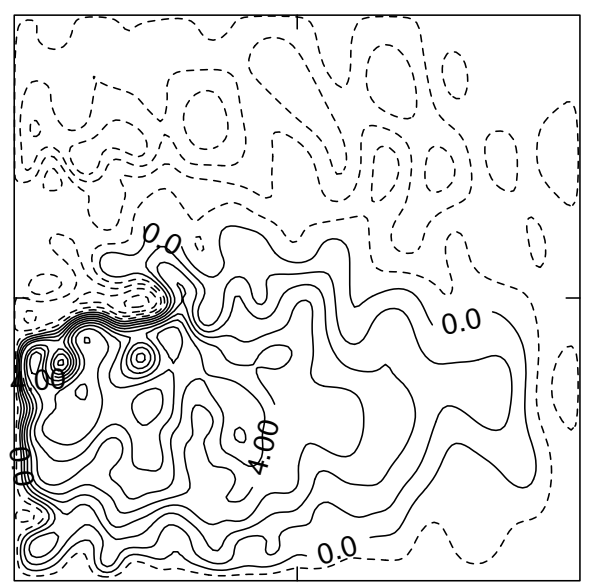

(d)

Figure 9: The upper-ocean, time-mean velocity streamfunctions, $\langle\bar{\psi}\rangle,\left(\mathrm{CI}=10^{4} \mathrm{~m}^{2} \mathrm{~s}^{-1}\right)$ and the corresponding divergences of the time-mean eddy flux, $\left\langle\mathbf{F}^{\prime}\right\rangle$, (color) that are obtained with the statistical decompositions (a) A and (b) B (see appendix B). The corresponding streamfunction snapshots, which are analogous to those in Figs. 1c and 3b, are shown in panels (c) and (d), respectively. The minimum and maximum values of the color plotted quantities are: (a) -25.3 and $18.4 \times 10^{-8} \mathrm{~s}^{-2},(\mathrm{~b})-23.5$ and $8.7 \times 10^{-8} \mathrm{~S}^{-2}$. 


\begin{tabular}{ccc}
\hline Full PV flux & Thickness flux & Reynolds stress \\
\hline$\left\langle F_{L E}^{\prime}\right\rangle: \Downarrow$, down, $\mp$ & $\left\langle B_{L E}^{\prime}\right\rangle: \Downarrow$, down, $\mp$ & $\left\langle R_{L E}^{\prime}\right\rangle: \Downarrow$, down, \pm \\
\hline$\left\langle F_{E L}^{\prime}\right\rangle: \Uparrow$, up, ... & $\left\langle B_{E L}^{\prime}\right\rangle: \Uparrow$, up, \pm & $\left\langle R_{E L}^{\prime}\right\rangle: \Uparrow$, up, $\mp$ \\
\hline$\left\langle F_{E E}^{\prime}\right\rangle: \Uparrow$, up, $\mp$ & $\left\langle B_{E E}^{\prime}\right\rangle: \Uparrow$, up, $\mp$ & $\left\langle R_{E E}^{\prime}\right\rangle: \ldots, \ldots, \ldots$ \\
\hline$\left\langle F^{\prime}\right\rangle: \Uparrow$, up, $\mp$ & $\left\langle B^{\prime}\right\rangle: \Uparrow$, up, $\mp$ & $\left\langle R^{\prime}\right\rangle: \ldots, \ldots, \ldots$. \\
\hline
\end{tabular}

Table 1: Meridional patterns of the upper-ocean eddy fluxes and their components across the subtropical EJ. Symbols $\Uparrow$ and $\Downarrow$ indicate northward and southward directions of the flux, respectively; symbols up and down indicate whether the fluxes are locally up- or down-gradient with respect to the time-mean absolute $\mathrm{PV},\langle\bar{Q}\rangle$; symbols \pm and $\mp$ indicate the alternatingsign eddy flux divergence pattern that enhances (i.e., positive to the north of and negative to the south of the EJ axis) or weakens (i.e., the opposite configuration) the eastward flow in the jet. The multiple-dot symbol, ...., is applied when the corresponding pattern can not be described in terms of a simple meridional structure. 


\begin{tabular}{ccc}
\hline Full PV flux & Thickness flux & Reynolds stress \\
\hline$\left\langle F_{L E}^{\prime}\right\rangle: \Downarrow$, down, $\mp$ & $\left\langle B_{L E}^{\prime}\right\rangle: \ldots, \ldots, \ldots$ & $\left\langle R_{L E}^{\prime}\right\rangle: \Downarrow$, down, \pm \\
\hline$\left\langle F_{E L}^{\prime}\right\rangle: \Uparrow$, up, \pm & $\left\langle B_{E L}^{\prime}\right\rangle: \Downarrow$, down, \pm & $\left\langle R_{E L}^{\prime}\right\rangle: \Uparrow$, up, $\mp$ \\
\hline$\left\langle F_{E E}^{\prime}\right\rangle: \Downarrow$, down, ... & $\left\langle B_{E E}^{\prime}\right\rangle: \Downarrow$, down, \pm & $\left\langle R_{E E}^{\prime}\right\rangle: \Uparrow$, up, $\mp$ \\
\hline$\left\langle F^{\prime}\right\rangle: \Downarrow$, down, \pm & $\left\langle B^{\prime}\right\rangle: \Downarrow$, down, \pm & $\left\langle R^{\prime}\right\rangle: \Uparrow$, up, $\mp$ \\
\hline
\end{tabular}

Table 2: The same as in Table 1, but for the deep ocean. 